\title{
Flèches du temps et de l'espace : une compréhension du second principe de la thermodynamique
}

\author{
Time and space arrows: an understanding of the second law of \\ thermodynamics
}

\author{
Bernard Guy ${ }^{1}$ \\ ${ }^{1}$ Mines Saint-Etienne, Institut Mines Télécom, France, guy@emse.fr
}

RÉSUMÉ. Les formulations courantes du second principe de la thermodynamique lient entropie et temps de façon quasi-exclusive : "l'entropie d'un système isolé augmente avec le temps ". Et ceci, que ce l'on se rapporte à la formulation historique (Carnot, Clausius) ou statistique (Boltzmann, Gibbs). Cette présentation ne facilite pas une compréhension intuitive de l'entropie. De plus, elle ne permet pas de voir l'unité conceptuelle qui se cache derrière deux contenus très différents, l'un portant sur des quantités de chaleur divisées par des températures, l'autre sur un nombre d'états microscopiques assurant un même état macroscopique. Ces difficultés s'allègent à condition d'insister sur le rôle de la variable spatiale et ses gradients. Ainsi les deux formulations peuvent être reprises dans ce sens, la première en disant que " la chaleur va naturellement du chaud vers le froid », et la seconde en disant qu' " un système hétérogène isolé évolue de façon plus probable vers l'homogénéité ». On voit ainsi l'unité conceptuelle du second principe : il exprime que, dans un système isolé, des hétérogénéités, qu'elles soient de température ou d'autres paramètres, ont tendance à s'adoucir. Diverses questions sont examinées dans le même cadre : la notion d'équilibre, la question des échelles de temps et d'espace, la distinction entre travail et chaleur, le problème du temps etc.

ABSTRACT. The common formulations of the second law of thermodynamics link entropy and time in an almost exclusive way: "the entropy of an isolated system increases with time". This applies to both historical (Carnot, Clausius) and statistical (Boltzmann, Gibbs) formulations. This presentation does not facilitate an intuitive understanding of the entropy. Moreover, it does not allow us to see the conceptual unity behind two very different contents, one dealing with quantities of heat divided by temperatures, the other with a number of microscopic states ensuring the same macroscopic state. These difficulties are alleviated if we insist on the role of the spatial variable and its gradients. Thus the two usual formulations can be repeated in this sense, the first by saying that "heat naturally goes from hot to cold", and the second by saying that "an isolated heterogeneous system evolves in a more probable way towards homogeneity". This shows the conceptual unity of the second law: it expresses that, in an isolated system, heterogeneities, whether of temperature or other parameters, tend to decrease. Various topics are examined in the same frame: the notion of equilibrium, the question of time and space scales, the distinction between work and heat, the time problem etc.

MOTS-CLÉS. thermodynamique, second principe, quantité de chaleur, probabilité, échelle microscopique, échelle macroscopique, flèche du temps, flèche de l'espace, homogénéité, température.

KEYWORDS. thermodynamics, second law, quantity of heat, probability, microscopic scale, macroscopic scale, time arrow, space arrow, homogeneity, temperature.

\section{Introduction}

La thermodynamique reste une science difficile à comprendre et à enseigner (voir par exemple [GON 08]. Si elle fournit des règles utiles aussi bien à l'ingénieur qu'au chercheur, le support de l'intuition fait souvent défaut pour se représenter ses concepts de base ; cela est vrai en particulier pour le second principe. Dans cet article, nous nous proposons de fournir des images, que nous espérons parlantes, pour une approche de la thermodynamique. Elles sont fondées sur l'étude qualitative d'exemples dont nous ne prétendons pas pour l'instant la valeur générale. Il conviendra dans le futur de compléter la trame proposée par l'examen de davantage d'aspects de la théorie, accompagnés des détails des formulations mathématiques. Dans la foulée, nous discutons, selon 
notre cadre de compréhension, ce que l'on appelle « le problème du temps » en thermodynamique : comment rendre compte de l'irréversibilité des lois de la thermodynamique à partir des lois réversibles de la mécanique ? Nous ne prétendons pas faire preuve d'originalité, n'ayant pas la connaissance exhaustive de la matière se rapportant au sujet; nous souhaitons d'abord mettre en forme notre propre façon de voir les choses, et fournir au lecteur les représentations qui nous ont manqué lorsque nous avons-nous-même appris la thermodynamique. Nous pensons en particulier (première partie) que la clé pour avancer dans la compréhension de ce domaine de la science est de redonner toute leur place aux variables d'espace et à leur couplage avec la variable temporelle, cette dernière ne devant pas (plus) être envisagée isolément ${ }^{1}$ (voir nos travaux par ailleurs, par exemple [GUY 11a] [GUY 11c] [GUY 16] [GUY 17]). D’une façon plus large, cet article discute quelques aspects de la thermodynamique à la lumière de notre compréhension des concepts d'espace et de temps (seconde partie) : on doit toujours les appréhender ensemble, dans le cadre d'une pensée relationnelle $^{2}$ (les relations spatiales et les relations temporelles sont les mêmes relations ; cf. [GUY 13b] [GUY 14]).

\section{Première partie : flèches du temps et de l'espace}

\section{Deux expressions du second principe de la thermodynamique (rappels)}

\subsection{Formulation historique avec quantités de chaleur et températures (Carnot, Clausius)}

Nous passons sous silence le premier principe et la conservation de l'énergie ; l'étudiant de la thermodynamique peut plus ou moins s'y retrouver, encore que la notion d'énergie interne et de transfert de quantité de chaleur méritent de l'attention. Par contre, comme nous l'avons dit, on est davantage désarmé pour se faire une image du second principe.

La formulation historique (macroscopique) $\mathrm{du}$ second principe reste souvent la première enseignée et discute les échanges de quantité de chaleur et de travail entre un système et son environnement. Dans l'écriture due à Clausius [CLA 64] à partir des travaux de Carnot [CAR 24] on désigne par $\mathrm{Q}_{\mathrm{i}}$ les quantités de chaleur échangées par le système avec des réservoirs extérieurs de températures constantes $T_{i}$. Le second principe stipule que la somme des quantités $Q_{i} / T_{i}$ correspondantes est nulle pour un cycle (le système parcourt une série de transformations le ramenant à son état initial); on doit préciser (le sens de cette condition est éclairé par la suite) que ce cycle se fait par des transformations proches de l'équilibre, ou encore sans pertes ni dissipations internes. Ceci s'écrit :

$$
\delta S=\oint \frac{\delta Q}{T}=0
$$

Où l'on définit une fonction d'état $\mathrm{S}$, l'entropie. Les échanges se font avec l'extérieur du système et il peut être utile de préciser les notations et remplacer $\delta S$ par $\delta_{\mathrm{e}} \mathrm{S}$, avec l'indice e comme extérieur ${ }^{3}$.

Lorsque des dissipations ${ }^{4}$ internes interviennent, on fait apparaître un terme supplémentaire, positif ou nul, nommé $\delta_{\mathrm{i}} \mathrm{S}$ (où l'indice i signifie intérieur). Nous envisageons des petites variations

\footnotetext{
${ }^{1}$ Il s'agit au moins de rendre explicites des formulations qui ne le sont pas.

${ }^{2}$ Dans la pensée relationnelle, à laquelle nous ferons appel en quelques lieux de cet article, les entités sont définies par opposition / comparaison les unes avec les autres; alors que dans la pensée substantielle (plus habituelle) on attribue à chaque entité des qualités qui lui sont propres, sans besoin de regarder les autres entités pour ce faire [Guy 11a].
}

${ }^{3}$ On pourrait mettre le même indice pour $\delta Q$.

${ }^{4}$ La définition d'une dissipation n'est pas forcément évidente comme on le discute plus loin. 
$\delta$; l'entropie étant une fonction d'état, on peut aussi bien écrire des différentielles exactes $\mathrm{d}$, ce qui n'est pas le cas en toute rigueur pour les quantités de chaleur ; le terme supplémentaire s'écrit :

$$
d_{i} S=\sum_{i=1}^{n} \frac{d \pi_{i}}{T_{i}}+\sum_{(i, j)}\left(\frac{d Q_{j i}}{T_{i}}+\frac{d Q_{i j}}{T_{j}}\right) \geq 0
$$

Pour un système constitué d'un ensemble de corps $C_{i}(i=1 \ldots n)$ de températures uniformes $T_{i} \cdot Q_{i j}$ désigne la quantité de chaleur absorbée par $C_{i}$ en provenance de $C_{j}$, et $Q_{j i}$ désigne la quantité de chaleur absorbée par $C_{j}$ en provenance de $C_{i}$. On a par construction $\mathrm{dQ}_{\mathrm{ij}}=-\mathrm{dQ}_{\mathrm{ji}}$. La sommation $(\mathrm{i}, \mathrm{j})$ est étendue à toutes les combinaisons 2 à 2 des corps du système. On a donc :

$$
d_{i} S=\sum_{i=1}^{n} \frac{d \pi_{i}}{T_{i}}+\sum_{(i, j)}\left(\frac{1}{T_{i}}-\frac{1}{T_{j}}\right) d Q_{i j} \geq 0
$$

On a fait une distinction (utile par la suite) entre les échanges de chaleur internes et des phénomènes non précisés pour l'instant, englobés dans le «travail non compensé » $\pi\left(\mathrm{d} \pi_{\mathrm{i}}\right.$ est le travail non compensé afférent au corps $\mathrm{C}_{\mathrm{i}}$ ). La forme du terme relatif aux échanges de chaleur internes est la même que celle écrite pour les échanges externes dans (1), c'est-à-dire une somme de termes en dQ/T. L'expression précédente (2') est donnée dans Fer [FER 70].

A partir des deux volets synthétisés dans les équations (1), (2) et (2'), une formulation générale du second principe s'exprime ainsi : la variation d'entropie d'un système est la somme de deux termes

$$
\mathrm{d} S=\mathrm{d}_{\mathrm{e}} \mathrm{S}+\mathrm{d}_{\mathrm{i}} \mathrm{S}
$$

Le premier résulte des échanges avec l'extérieur du système :

$$
\delta_{e} S=\int \frac{d Q_{e}}{T}
$$

dans le cas où l'on a uniquement des échanges de chaleur (définition historique); d'autres termes peuvent se rajouter dans ce que l'on appelle aussi le «flux » d'entropie; le signe de $\mathrm{d}_{\mathrm{e}} \mathrm{S}$ est quelconque et dépend des échanges. Le second terme dans (3) est donné par l'expression (2') cidessus. Le travail non compensé peut être développé en divers termes (voir traités de thermodynamique). Le cœur du second principe s'exprime par la condition que $\mathrm{d}_{\mathrm{i}} \mathrm{S}$ est positif ou nul. Pour un système isolé $d_{\mathrm{e}} \mathrm{S}$ est nul et il reste $\mathrm{d}_{\mathrm{i}} \mathrm{S} \geq 0$. Pour un cycle et dans le cas d'échanges uniquement thermiques dS est nul et s'il y a dissipation on a simultanément $\mathrm{d}_{\mathrm{i}} \mathrm{S} \geq 0$ et $\mathrm{d}_{\mathrm{e}} \mathrm{S} \leq 0$.

\subsection{Formulation probabiliste (Boltzmann, Gibbs)}

L'approche historique précédente permet de définir et mesurer l'entropie macroscopique par des expériences calorimétriques, à condition de minimiser les dissipations par d'autres phénomènes (voir la discussion précédente). Elle a été suivie par une autre approche dite statistique. On se rapporte aux travaux de Boltzmann [BOL 95] [BOL 98], repris par Gibbs [GIB 02], qui ont étudié les relations entre les phénomènes à l'échelle des particules microscopiques constituant le système et ceux du système global macroscopique ${ }^{5}$. Nous ne rediscutons pas la notion d'ensembles statistiques (Gibbs) ni les jeux d'hypothèses les définissant (ensembles micro-canonique, canonique et macro-canonique), nous plaçant dans le cadre des hypothèses de l'ensemble micro-canonique. Il correspond aux systèmes isolés étudiés précédemment ; il est exemplaire pour notre propos et nous place au cœur et au départ de l'approche statistique. Soit $\Omega$ le nombre de configurations

\footnotetext{
${ }^{5}$ On trouvera par exemple dans Barberousse [BAR 02], Jancovici [JAN 96], Richet [RIC 00] des informations sur l'approche statistique de la thermodynamique. Le Coze [LEC 10] discute le passage du microscopique au macroscopique et des hypothèses cachées qui le permettent (cf. notre analyse dans [GUY 11b], [GUY 13a]).
} 
microscopiques, ou complexions, donnant un même état macroscopique. La probabilité d'obtenir un système macroscopique donné est d'autant plus grande que le nombre $\Omega$ est grand; si tous les micro-états sont également probables (hypothèse de base de la physique statistique pour les systèmes isolés en équilibre), on peut définir l'entropie par

$$
\mathrm{S}=\mathrm{k}_{\mathrm{B}} \operatorname{Ln} \Omega
$$

Où $\mathrm{k}_{\mathrm{B}}$ est la constante de Boltzmann. Cette expression a été donnée par Boltzmann lui-même. L'expression du second principe proposée par Gibbs à la suite des travaux de Boltzmann exprime que, pour un système isolé, l'entropie dans son expression statistique donnée par (5) (c'est un $d_{i} S$ selon la notation de la section précédente) ne peut que croître, c'est-à-dire que le système tend vers des états plus probables ${ }^{6}$.

\subsection{Première discussion}

Deux définitions très différentes de l'entropie viennent d'être données: la première faisant intervenir des quantités de chaleur et des températures, la seconde des dénombrements de complexions; et deux formulations également très différentes du second principe, portant sur le signe de la dérivée temporelle de la grandeur entropie définie dans chacun des deux cas. La question qui se pose à tout étudiant de la thermodynamique est double :

a) quelle image se faire de chacune de ces présentations, sur des échanges de quantités de chaleur divisées par des températures d'une part, et sur des configurations microscopiques d'autre part ? Et :

b) y-a-t-il un seul concept physique derrière ces deux visions différentes dont on prononce l'équivalence ? C'est-à-dire, par quelle image unique comprendre l'équivalence (l'identité ?) entre ces deux représentations?

Si dans les traités de thermodynamique, on trouve (rarement) des éléments de réponse à la première question, on n'en trouve pas pour la seconde. Les deux formalismes, le formalisme historique (ou phénoménologique) et le formalisme statistique sont développés séparément. Une fois qu'on a obtenu des expressions de l'entropie par chacune des deux voies, on fait des identifications mathématiques entre elles et on en tire de nouvelles relations. Dans la suite du texte, nous voulons montrer comment relier de façon plus étroite, au moins au niveau conceptuel, les deux grandes formulations rappelées.

\subsection{Propriété d'extensivité}

Avant d'aller plus loin, rappelons que les expressions que nous avons données de l'entropie et du second principe ne sont pas valables pour n'importe quels types de systèmes; elles le sont pour ceux possédant la propriété dite d'extensivité ou additivité, stipulant qu'un certain nombre de propriétés du système sont en proportion de celles de sous-systèmes de tailles arbitraires que l'on peut y définir. En particulier l'entropie du système global est la somme de celles de ses parties; c'est ce qui permet d'ajouter les ratios $\mathrm{Q}_{\mathrm{i}} / \mathrm{T}_{\mathrm{i}}$ dans l'expression historique de l'entropie, ou d'obtenir l'expression $\mathrm{S}$ $=\mathrm{k}_{\mathrm{B}} \mathrm{Ln} \Omega$ dans l'approche statistique. Cette propriété exprime aussi l'indépendance des probabilités calculées pour différents sous-systèmes. Elle n'est pas possédée par les systèmes où s'exercent des forces à longue distance (voir par exemple [TSA 95]).

\footnotetext{
${ }^{6}$ Boltzmann l'avait dit aussi lui-même. Pour l'ensemble macro-canonique, l'entropie est définie par $S=\sum p_{i} \ln \left(p_{i}\right)$ où la sommation s'entend à tous les états $i$ de probabilités $p_{i}$; cette expression se généralise au cas continu en $S=\int$ plnpdp.
} 


\section{Sens des transferts thermiques (formulation historique)}

Avant de relier les deux formulations, reprenons tout d'abord la formulation historique et mettons en valeur une façon particulière de l'envisager. Celle-ci, proposée par Clausius lui-même, est généralement passée sous silence; F. Fer [FER 70] la commente en relation avec les autres présentations. On peut la résumer en disant : la chaleur ne peut aller spontanément que du chaud vers le froid. Ce principe se rapporte à des systèmes lieu de transferts thermiques (sans prise en compte d'autres sources d'irréversibilité ${ }^{7}$ ) et produisant éventuellement du travail (comme les machines à vapeur étudiées par Carnot).

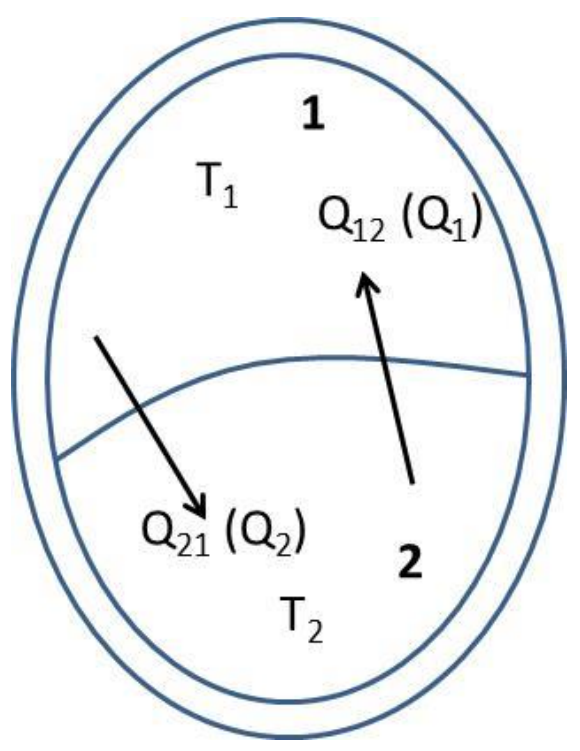

Figure 1. Le système étudié est composé de deux compartiments 1 et 2 aux températures $T 1$ et $T 2$, avec $T 2$ > T1. Ces deux compartiments échangent les quantités de chaleur Q21 $=Q 2$ (reçu par 2 en provenance

de 1) et Q12 = Q1 (reçu par 1 en provenance de 2). L'isolement du système impose Q1 + Q2 = 0, ou encore $Q 21=-Q 12$. Ces diverses conditions entraînent la positivité de la variation de la fonction entropie.

\subsection{Transferts de chaleur internes au système}

Ce principe peut être obtenu par (au moins) trois voies présentées dans les sections 2.1 à 2.3 . Examinons d'abord le terme $\mathrm{d}_{\mathrm{i}} \mathrm{S}$ de tout à l'heure dans l'expression (2'). Le terme en d $\pi$ est nul (il n'y a que des transferts thermiques) et il reste la condition :

$$
d_{i} S=\sum_{(i, j)}\left(\frac{1}{T_{i}}-\frac{1}{T_{j}}\right) d Q_{i j} \geq 0
$$

où les différents termes ont été définis plus haut. Pour la simplicité de l'exposé, considérons un système isolé composé de deux corps $C_{1}$ et $C_{2}$ seulement, aux températures $T_{1}$ et $T_{2}$ (Fig. 1). Soit $T_{2}$ $>\mathrm{T}_{1}$. Appelons $\mathrm{Q}_{1}$ la chaleur reçue par $\mathrm{C}_{1}$ en provenance de $\mathrm{C}_{2}$ (soit $\mathrm{Q}_{12}$ dans la notation donnée plus haut) et $\mathrm{Q}_{2}$ la chaleur reçue par $C_{2}$ en provenance de $C_{1}\left(\right.$ ou $\left.Q_{21}\right)$. On a ici $Q_{1}=-Q_{2}$ par définition, qui exprime aussi la condition d'isolement du système ; (6) s'écrit alors

$$
d_{i} S=\left(\frac{1}{T_{1}}-\frac{1}{T_{2}}\right) Q_{1} \geq 0
$$

Comme le terme en températures est positif, car $\mathrm{T}_{1}<\mathrm{T}_{2}$, la contrainte portant sur $\mathrm{d}_{\mathrm{i}} \mathrm{S}$ impose que $\mathrm{Q}_{1}$ soit positif ; c'est-à-dire que la chaleur reçue par $\mathrm{C}_{1}$ est positive et celle reçue par $\mathrm{C}_{2}$ est

\footnotetext{
${ }^{7}$ On se limite dans ce qui suit à des échanges thermiques. Cela ne nuit pas à la généralité de l'expression de l'entropie comme on le discute plus loin.
} 
négative ; en d'autres termes, la chaleur passe bien du corps chaud à la température $\mathrm{T}_{2}$ vers le corps plus froid à la température $T_{1}$. La réciproque est également vraie, c'est-à-dire que si $T_{2}>T_{1}$ et si $Q_{1}$ est positif, c'est-à-dire si la chaleur va du chaud vers le froid, alors le $\mathrm{d}_{\mathrm{i}} \mathrm{S}$ écrit dans la relation (7) est bien positif.

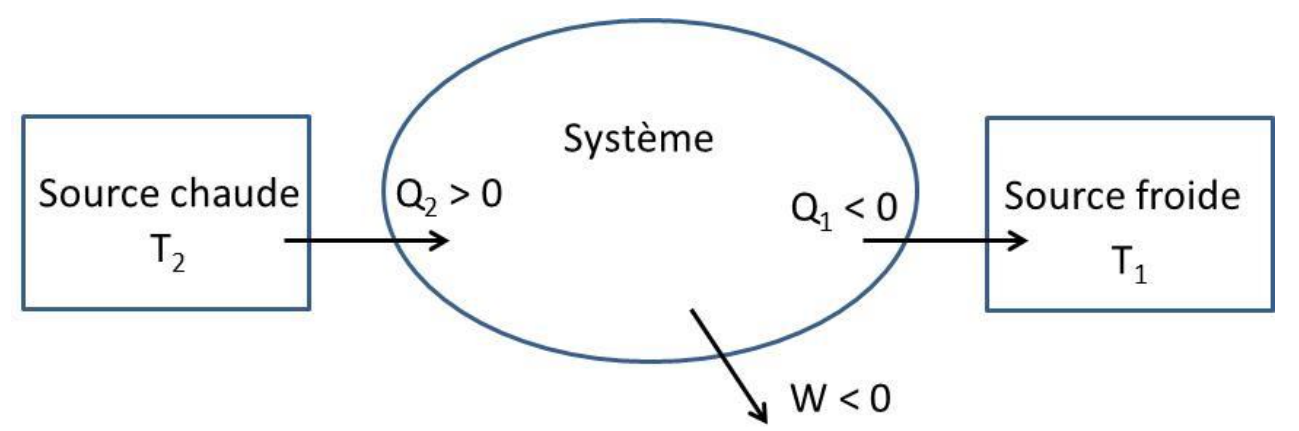

Figure 2. Système échangeant avec deux sources externes et produisant du travail. La condition de transfert global de la chaleur depuis la source chaude vers la source froide et la condition du caractère négatif ou nul de la variation de la fonction entropie sont équivalentes.

\subsection{Cycles dithermes produisant du travail}

Dans le cas de cycles dithermes produisant du travail sans pertes internes, la condition de type (1) portant sur le terme $d_{\mathrm{e}} \mathrm{S}$ régit aussi le passage d'une quantité de chaleur de la source chaude à la source froide (Fig. 2). Soient $\mathrm{Q}_{1}$ et $\mathrm{Q}_{2}$ les quantités de chaleur échangées par le système respectivement avec la source froide à la température $\mathrm{T}_{1}$ et la source chaude à la température $\mathrm{T}_{2}$ (les quantités de chaleur sont comptées positivement pour un apport au système et négativement dans le cas contraire; les notations ont des signes différents de ceux adoptés à l'instant dans l'étude des transferts purement internes car on se réfère au système dans son ensemble, et non à l'un ou l'autre de ses compartiments). Nous ne reprenons pas la discussion de Carnot stipulant qu'il faut deux températures différentes pour produire du travail W. La conservation de l'énergie s'écrit

$$
\mathrm{Q}_{1}+\mathrm{Q}_{2}+\mathrm{W}=0
$$

Nous nous situons dans la situation «naturelle » où le système peut fournir du travail à l'extérieur, c'est-à-dire que $\mathrm{W}<0$ (par opposition, si l'on doit fournir du travail au système, le transfert thermique ne respectera pas la condition que nous voulons mettre en évidence). On a donc

$$
\mathrm{Q}_{1}+\mathrm{Q}_{2}=-\mathrm{W}>0
$$

On a de plus la condition énoncée par Carnot et Clausius pour un cycle :

$$
\mathrm{Q}_{1} / \mathrm{T}_{1}+\mathrm{Q}_{2} / \mathrm{T}_{2}=0
$$

Ou encore

$$
\frac{Q_{1}}{Q_{2}}=-\frac{T_{1}}{T_{2}}
$$

Ceci entraîne, $\mathrm{T}_{1}$ et $\mathrm{T}_{2}$ étant positifs, que $\mathrm{Q}_{1}$ et $\mathrm{Q}_{2}$ sont de signe contraire. L'expression précédente peut aussi s'écrire 
$\frac{Q_{1}+Q_{2}}{Q_{2}}=\frac{T_{2}-T_{1}}{T_{2}}>0$

$\mathrm{Q}_{2}$ est donc du même signe que $\mathrm{Q}_{1}+\mathrm{Q}_{2}$ c'est-à-dire positif, alors que $\mathrm{Q}_{1}$ est négatif. C'est dire qu'au total le système reçoit de la chaleur de la source chaude et donne de la chaleur à la source froide ; ou encore le transfert global de chaleur se fait de la source chaude vers la source froide (une partie de cette chaleur peut être distraite en travail) ; c'est ce que nous cherchions à vérifier.

\section{Réciproque}

On peut écrire une réciproque de la façon suivante. Soit un système en communication avec deux réservoirs de températures $T_{2}$ et $T_{1}$ tels que $T_{2}>T_{1}$. On a donc

$$
\frac{T_{2}-T_{1}}{T_{2}}>0
$$

Ce système échange des quantités de chaleur $\mathrm{Q}_{2}$ et $\mathrm{Q}_{1}$ avec ces réservoirs, et le transfert respecte la condition que la chaleur va du chaud vers le froid; c'est-à-dire que l'on a $\mathrm{Q}_{2}>0$ et $\mathrm{Q}_{1}<0$. Ce système peut produire du travail $\mathrm{W}$, et on a donc $\mathrm{W}<0$; la conservation de l'énergie s'écrit

$$
\mathrm{Q}_{1}+\mathrm{Q}_{2}=-\mathrm{W}>0
$$

On tire de (13) et (14) que

$$
\frac{Q_{1}+Q_{2}}{Q_{2}}>0
$$

On vérifie que les conditions de signe portant sur les relations (13) et (15) sont remplies simultanément, les deux quantités étant positives en même temps. On peut donc relier les deux expressions (13) ou (15), chacune dans une fonction monotone de l'autre passant par l'origine. On peut décider d'identifier les deux expressions, ce qui définit une échelle de température en relation avec les échanges de chaleur:

$$
\frac{Q_{1}+Q_{2}}{Q_{2}}=\frac{T_{2}-T_{1}}{T_{2}}
$$

D'où on tire

$$
\frac{Q_{1}}{Q_{2}}=-\frac{T_{1}}{T_{2}}
$$

Ainsi que

$$
\sum \frac{Q_{i}}{T_{i}}=0
$$

Ce qui démontre la réciproque ; ceci est valable pour un cycle, le bilan énergétique total du système étant nul. Le ratio des quantités de chaleur échangées est imposé par le ratio des températures. Les grandeurs absolues ne sont pas connues. Suivant la valeur du travail fourni à l'extérieur, les grandeurs sont différentes et respectent la conservation de l'énergie dans (14). 


\subsection{Production d'entropie}

On peut enfin discuter le sens des transferts thermiques en utilisant l'écriture de ce que l'on appelle la production d'entropie. Il s'agit d'une formulation postérieure aux formulations historiques de Carnot et Clausius que l'on trouvera par exemple dans Glansdorff et Prigogine [GLA 71]. Dans un système où il n'y a que des transferts thermiques, on a :

$$
\mathrm{P}(\mathrm{S})=\int \mathrm{D}_{\mathrm{th}}(\nabla \mathrm{T})^{2}=\int \mathrm{D}_{\mathrm{th}}(\partial \mathrm{T} / \partial \mathrm{x})^{2}>0
$$

où $\mathrm{P}(\mathrm{S})$ est la production d'entropie (c'est-à-dire $\mathrm{d}_{\mathrm{i}} \mathrm{S} / \mathrm{dt}$ ), $\mathrm{D}_{\text {th }}$ le coefficient de diffusivité thermique et $\nabla \mathrm{T}=\partial \mathrm{T} / \partial \mathrm{x}$ le gradient de température, où l'on se limite pour la simplicité de l'écriture à une dimension d'espace $\mathrm{x} . \mathrm{P}(\mathrm{S})$ est positive ou nulle, selon le second principe. Le gradient intervient au carré, et il y a donc équivalence entre $\mathrm{P}(\mathrm{S})$ positif ou nul et $\mathrm{D}_{\text {th }}$ positif ou nul ; ceci exprime une nouvelle fois que la chaleur va du chaud vers le froid. La réciproque exprime que le sens d'écoulement de la chaleur du chaud vers le froid (positivité de $\mathrm{D}_{\text {th }}$ ) entraîne une production d'entropie positive, ce qui est une expression du second principe.

\subsection{Une formulation du second principe}

Reprenant ce que nous avons écrit au début de la présente section 2, on pourra exprimer le second principe de la façon suivante:

Pour des systèmes qui sont le lieu d'échanges thermiques, la chaleur va spontanément du chaud vers le froid ; cela est valable pour les systèmes isolés et leurs sous parties internes ; cela est aussi valable pour les systèmes échangeant de la chaleur avec l'extérieur et pouvant produire du travail. Si l'on appelle $Q_{i}$ les échanges de chaleur avec des réservoirs de températures $T_{i}$, la condition précédente s'exprime à l'aide de la fonction $\Sigma \mathrm{Q}_{\mathrm{i}} / \mathrm{T}_{\mathrm{i}}$. Pour un cycle, cette fonction est négative ou nulle.

Nous considérerons pour l'instant que nous avons ici une forme équivalente à l'une des expressions historiques du second principe ; un travail rigoureux de mathématiques serait nécessaire pour s'assurer de la stricte équivalence entre la formulation précédente et telle ou telle formulation du second principe, sachant que, selon Fer (op. cit.), toutes ne sont pas strictement équivalentes suivant les diverses hypothèses adoptées. La fonction $\mathrm{S}=\Sigma Q_{i} / T_{i}$ sur laquelle porte une condition de signe n'est sans doute pas la seule fonction mathématique des grandeurs $\left(Q_{1}, Q_{2}, T_{1}, T_{2}\right)$ par exemple dans le cas de deux sources, garantissant que le transfert thermique se fait dans le bon sens, c'est-àdire que $\mathrm{Q}_{2}>0$ et $\mathrm{Q}_{1}<0$ si $\mathrm{T}_{2}>\mathrm{T}_{1}$, mais nous n'en discuterons pas ${ }^{8}$.

\section{Retour sur les configurations microscopiques (approche statistique)}

Revenons à la seconde formulation du second principe de la thermodynamique présentée plus haut; elle porte sur l'évolution d'une fonction du nombre d'états microscopiques assurant un même état macroscopique, la valeur de cette fonction exprimant la probabilité des états macroscopiques (« le système -macroscopique- tend vers l'état le plus probable » c'est-à-dire celui correspondant au maximum d'états microscopiques possibles). Caractérisons cette situation par des configurations effectives dans l'espace. Prenons comme exemple (Fig. 3) un système comprenant un ensemble très grand d'atomes élémentaires supposés discernables pour les besoins du calcul de dénombrement ${ }^{9}$ et dénotés $1,2 \ldots$. Nous voulons étudier la multitude d'états microscopiques et leur attribuer des probabilités pour un même état macroscopique. A chaque disposition des $\mathrm{n}$ atomes, on peut calculer

\footnotetext{
${ }^{8}$ L'unicité n'est-elle pas imposée par le choix d'une échelle des températures ?

${ }^{9}$ Nous sommes donc dans le cas non quantique (où il y aurait indiscernabilité). 
une probabilité qui est proportionnelle au nombre de façons de disposer les $\mathrm{n}$ atomes pour avoir la même figure d'ensemble. Ce genre de calcul est fait dans la littérature et nous ne le reprendrons pas (voir par exemple[GON 08] : les atomes sont distribués sur un maillage rectangulaire, représentant par exemple un réseau cristallin ou des compartiments d'un réservoir de gaz). On vérifiera qu'une distribution dispersée où tous les atomes remplissent l'ensemble de l'espace de façon équilibrée (Fig. 3a) est beaucoup plus probable qu'une distribution inhomogène où apparaissent des ilots d'atomes plus rapprochés au sein de zones où ils sont au contraire moins nombreux (Fig. 3b). Les calculs reportés dans la littérature (par exemple Gonczi, op. cit.) montrent que la moindre inhomogénéité de remplissage des molécules d'un gaz entre simplement deux compartiments de taille égale, a une probabilité très notablement inférieure à celle du remplissage en quantités égales des deux compartiments. Ces figures visualisent ce que l'on appelle l'ordre et son lien avec l'aspect probabiliste : la figure $3 \mathrm{~b}$ représente une configuration plus ordonnée, à plus faible entropie, que la figure $3 \mathrm{a}$, plus désordonnée, à plus forte entropie ${ }^{10}$.
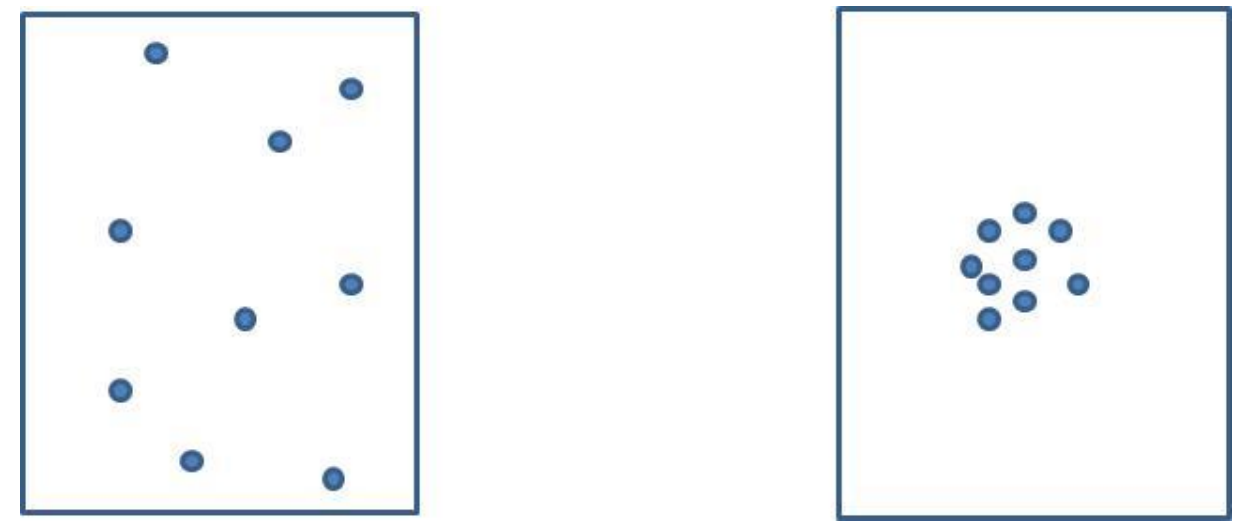

Figure 3 a et b. Deux dispositions des particules dans un système fermé. A gauche (Fig. 3a), les particules sont disposées de façon dispersée dans l'ensemble du système (état plus probable ou plus désordonné), alors qu'à droite (Fig. 3b), les particules sont disposées de façon concentrée (état plus improbable, ou plus ordonné).

Nous n'étudierons pas d'autres situations et considérerons de façon générale, au moins qualitativement, que les variations d'entropie correspondent à des variations de l'état plus ou moins inhomogène de la distribution des particules microscopiques dans l'espace (c'est l'entropie de configuration ; on discute plus loin le cas des entropies dites de vibration et de rotation).

\section{Le chemin vers l'état le plus probable}

Le second principe ne se contente pas de dénombrer les chances d'avoir tel ou tel état macroscopique en fonction du nombre de configurations microscopiques; il affirme que, pour des systèmes isolés, le système va effectivement se diriger vers l'état le plus probable. La question qui se pose alors est: pourquoi, à partir d'une configuration donnée, le système va-t-il aller spontanément vers l'état le plus probable ? Que veut dire «spontanément»? Dans l'esprit de notre recherche, nous voulons comprendre les choses en nous rapportant au déploiement du système dans l'espace. L'approche de Boltzmann porte sur la fonction de distribution statistique $\mathrm{f}(\mathrm{x}, \mathrm{v}, \mathrm{t}) \mathrm{des}$ vitesses et des positions des particules du système (étude d'un gaz) et son évolution dans le temps ; mais elle ne regarde pas les trajectoires elles-mêmes qui sont le support de cette évolution. Le théorème « $\mathrm{H}$ » de cet auteur indique qu'il existe une fonction de cette distribution qui augmente au

\footnotetext{
${ }^{10}$ Nous n'utiliserons pas cette notion d'ordre dans la suite ; elle ne fait pas spontanément penser à des gradients spatiaux (qui nous intéressent dans ce texte), mais on conviendra (sous réserve de définir une fonction adéquate portant sur le système et le quantifiant) qu'une disposition ordonnée fait apparaître davantage de gradients qu'une disposition désordonnée.
} 
cours des collisions des particules entre elles (cette approche concerne plus largement une famille d'équations non invariantes par renversement du temps $\mathrm{t} \rightarrow-\mathrm{t}$, conduisant toutes à des évolutions « irréversibles » : l'équation de Boltzmann, les équations maîtresses etc.). Les auteurs ont vu dans ce théorème la loi de croissance de l'entropie et ont procédé à une identification entre la fonction étudiée par Boltzmann et l'entropie définie par ailleurs. Malgré le bon fonctionnement de cette identification, cette approche ne correspond pas à ce que nous recherchons, car comme nous l'avons dit, les trajectoires individuelles des particules dans l'espace ne sont pas représentées ni discutées. Elles sont cachées dans la fonction de distribution qui est continue, c'est-à-dire s'applique à une population infinie de particules (limite thermodynamique). Comme il y a tant de particules, on abandonne l'idée que l'on pourrait considérer la trajectoire d'aucune ! Malgré le passage par les collisions, on ne comprend pas davantage pourquoi le système va au bout du compte se diriger vers l'état le plus probable. La fonction de distribution $f(x, v, t)$ ne fournit pas la probabilité d'une trajectoire. L'approche probabiliste apporte des progrès en terme de définitions de fonctions utiles mais on reste devant une énigme qui est celle dite du problème du temps, ainsi que Boltzmann l'admet d'ailleurs lui-même (voir aussi l'analyse de Bricmont [BRI 95]).

Proposons une approche différente en essayant de raisonner sur les trajectoires concrètes, dans une situation qui ne concerne d'abord qu'un tout petit nombre de particules. Ainsi, il faut considérer ce qui suit comme une simple proposition de recherche (dont nous reparlons dans la section 10.1 plus bas). Prenons un système de deux particules $\mathrm{A}$ et $\mathrm{B}$, et étudions le mouvement de l'une des deux, A par exemple, par rapport à la seconde B. Nous pouvons faire une partition entre les trajectoires qui vont regrouper $A$ et $B$, c'est-à-dire qui mènent $A$ vers $B$, et celles qui au bout du compte vont disperser $\mathrm{A}$ et $\mathrm{B}$, c'est à dire éloigner $\mathrm{A}$ de $\mathrm{B}$. Ce faisant, c'est par son but que nous qualifions une trajectoire. En comparant les diverses trajectoires les unes aux autres, on peut regarder les proportions ou probabilités de celles qui ont tendance à regrouper A et B (nous dirons aussi que ce sont celles qui rendent le système hétérogène) par rapport à celles qui ont tendance à disperser $\mathrm{A}$ et $\mathrm{B}$ (nous dirons que ce sont celles qui vont le rendre homogène ${ }^{11}$ ). En raccourci, nous attribuons une probabilité à une trajectoire en fonction de l'état qu'elle permet de rejoindre, par rapport à l'ensemble des états possibles et donc par rapport à l'ensemble des trajectoires possibles.

L'exemple est illustré sur la Figure 4. Regardons les divers chemins qui pourraient rapprocher A de B. Si B est vu par A sous un angle $\varepsilon$, sur les $360^{\circ}$ (en restant dans une discussion dans le plan et pour des chemins rectilignes ${ }^{12}$ ), la probabilité pour A d'aller vers B, regroupant ainsi A et B, est donc égale au rapport $\varepsilon / 360^{\circ}$ (c'est la proportion des directions des vitesses qui mènent $A$ vers $B$ ). Il est ainsi effectivement moins probable, ou plus improbable, à partir des conditions que nous nous sommes données, d'aller vers un état que nous avons qualifié d'hétérogène ${ }^{13}$.

Reprenons alors la conclusion obtenue dans la section précédente 3, stipulant qu'un état inhomogène (associant des zones «concentrées » et des zones dispersées) est moins probable qu'un état homogène. Nous venons à l'instant d'attribuer une probabilité moins grande à la trajectoire qui rejoint cet état. Nous observons donc une convergence entre la «probabilité » des trajectoires et celle des états, c'est-à-dire une correspondance entre trajectoires probables et états auxquels elles conduisent, c'est-à-dire états probables.

\footnotetext{
${ }^{11}$ On conviendra qu'il est un peu abusif de parler d'homogénéité ou d'hétérogénéité pour un système de deux particules ! Nous ne présentons là que la trame d'un raisonnement qui devra être étendu.

${ }^{12}$ C'est au moins le cas pour des portions élémentaires de déplacement.

${ }^{13}$ S'il y a plus de deux, soit n, points matériels à « rassembler », toutes choses égales par ailleurs (en particulier des distances initiales entre points susceptibles de se rassembler en gros égales), le ratio précédent $\varepsilon / 360^{\circ}$ sera élevé à la puissance n.
} 
Qu'est ce qui fait que l'on va suivre les trajectoires probables ? Les vrais systèmes sont soumis à toutes sortes de perturbations qui vont leur faire quitter les chemins improbables : on peut penser par exemple à des perturbations infimes d'origine gravitationnelle ou électromagnétique venues de parties de l'univers extérieures au système étudié ${ }^{14}$. On peut aussi invoquer l'incertitude fondamentale concernant les grandeurs physiques concernées (que ce soit dans le cadre classique, quantique, ou dans le cadre de notre compréhension des choses, celle affectant les repères mêmes d'espace-temps utilisés). On peut aussi invoquer les situations d'instabilité et de sensibilité aux conditions initiales (une infime variation des conditions initiales entraîne une évolution radicalement différente).

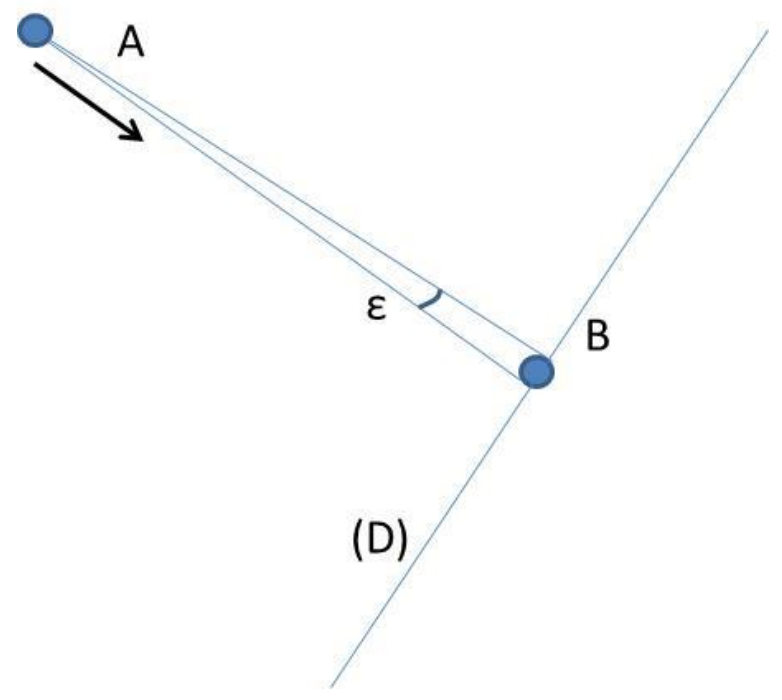

Figure 4. Système composé de deux particules $A$ et $B$. On cherche la vitesse initiale de $A$ permettant un rapprochement de $B$ : sa direction doit être comprise à l'intérieur d'un petit angle $\varepsilon$, faute de quoi les deux particules vont s'éloigner l'une de l'autre. La droite $(D)$ est perpendiculaire à la direction moyenne $A B$ (voir figure suivante).

On peut admettre que les perturbations affectant les trajectoires vont respecter des lois de nature probabiliste; reprenons l'exemple simple présenté à l'instant (la particule A se dirige vers la particule B) : on supposera ainsi que les perturbations ont autant de chances d'écarter la trajectoire vers sa droite que vers sa gauche par rapport à la droite reliant A à B (le raisonnement se fait dans le plan à deux dimensions); c'est-à-dire encore, il n'y a pas de biais dans un sens ou un autre (bruit blanc). C'est une situation analogue à l'approche probabiliste de la marche au hasard, de la diffusion ou du mouvement brownien. C'est encore une façon de dire que la loi des grands nombres opère : une somme de variations individuelles inconnues de même loi suit une répartition gaussienne. On peut représenter cela par une distribution de probabilité d'arriver en B depuis A, sachant que l'on vise $\mathrm{B}$ depuis $\mathrm{A}$ à l'instant initial (Fig. 5). Si la densité de probabilité présente au départ un pic resserré autour de B, celui-ci va s'étaler et avec le temps, c'est à dire les perturbations vont étaler la trajectoire également d'un côté et de l'autre du pic initial. Ceci va donc rendre de plus en plus improbable, par rapport à l'ensemble des trajectoires, celle permettant d'atteindre B.

Généralisons l'idée. Dans l'évolution, à partir de conditions initiales données, d'un système composé d'un grand nombre de particules, posons-nous la question de ce qui pourrait conduire à des états improbables, c'est-à-dire concentrés ? Réponse : il faudrait que les vitesses soient dirigées exactement dans la bonne (improbable) direction. Et si donc nous arrivons à régler ces conditions initiales de façon improbable, va-t-on pour autant aller vers les états improbables? Pendant un

\footnotetext{
${ }^{14}$ Ce qui montre la limite du qualificatif d' « isolé » que nous pouvons attribuer à tel système thermodynamique.
} 
certain temps, oui ; des simulations numériques montrent d'ailleurs que, en renversant les vitesses d'un système évoluant à partir de conditions concentrées, on le fait provisoirement revenir vers ces états (l'entropie décroitt ${ }^{15}$ ). Mais les simulations montrent que l'on va dériver et revenir à une évolution «normale» dispersante qui fait augmenter l'entropie. Pourquoi cela ? Au niveau numérique, la cause de ce phénomène est à rechercher dans les troncations des valeurs des grandeurs qui nous font quitter les chemins improbables, situation que nous considérerons pour l'instant en correspondance avec l'effet des perturbations et incertitudes dont nous venons de parler.

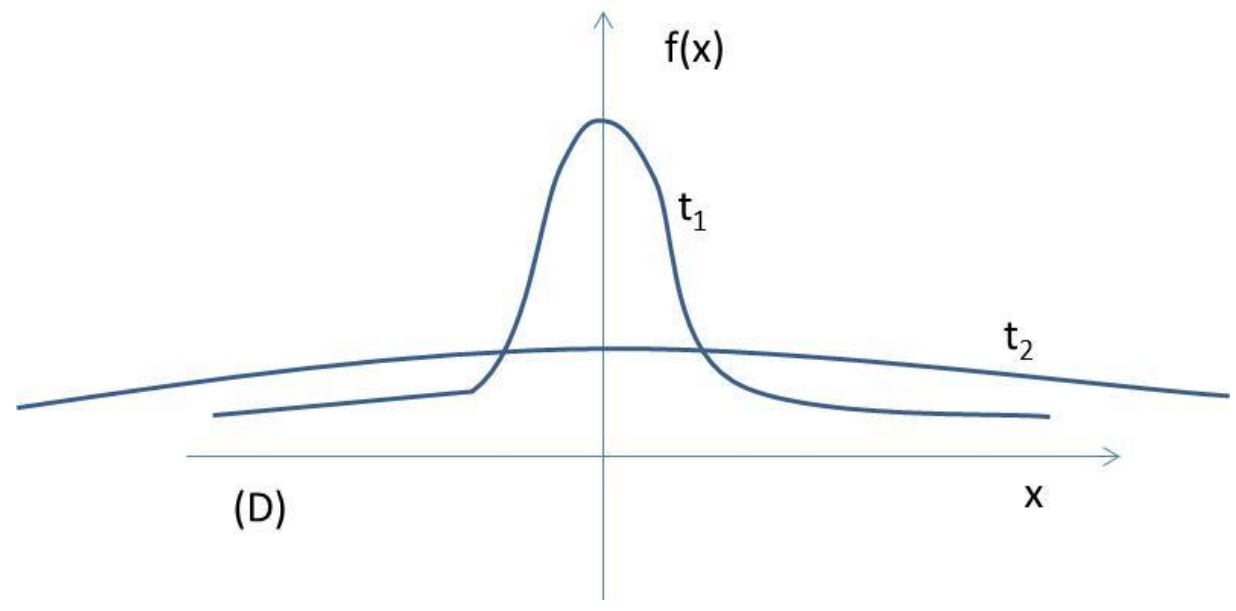

Figure 5. Distribution de probabilité pour la particule $A$ d'atteindre une position $x$ le long de la droite $(D)$ de la figure précédente, perpendiculaire à sa vitesse initiale ( $A$ vise $B$ correspondant à $x=0)$. S'il n'y a pas de perturbation de la trajectoire, on obtient la fonction désignée par 11 ( $A$ va très probablement atteindre $B$ ).

Mais si la trajectoire est perturbée, selon de petits angles également distribués de part et d'autre de la direction $A B$, la fonction va s'étaler, et ce d'autant plus que les perturbations se rajoutent au cours du temps (forme désignée par 12 ). La probabilité pour $A$ d'atteindre $B f(x) d x$ autour de $x=0$ (pour un petit $d x$ correspondant à l'angle $\varepsilon$ défini Fig. 4) va devenir infime par rapport à la probabilité de l'ensemble des autres positions. L'évolution " naturelle " du système (soumis à de telles perturbations) ne favorise pas le rapprochement de $A$ et $B$ malgré la valeur de la vitesse initiale.

Nous ne creuserons pas davantage et considérerons pour l'instant, de façon utile pour notre propos, que cette convergence a un sens plus général et que la corrélation entre états probables et trajectoires probables est une sorte de bijection. Le dire procède d'une pensée relationnelle qui ne veut pas dissocier une position des chemins qui y conduisent et donnent son sens même à une position. Un important travail conceptuel et mathématique serait utile pour le préciser ; considéronsle comme piste de recherche avant mieux. Cela devra permettre le lien formel entre les évolutions d'un système macroscopique décrit par des expressions en sommes de $\mathrm{Q}_{\mathrm{i}} / \mathrm{T}_{\mathrm{i}}$ et celles d'un système décrit au niveau microscopique par les sommes de $\mathrm{k}_{\mathrm{b}} \mathrm{Ln} \Omega$. Les trajectoires et leurs probabilités devront être calculées pour des transferts de chaleur dans un cas, et pour telle ou telle distribution des concentrations d'un mélange dans l'autre (un auteur tel Ken A. Dill, [DIL 08], se pose de façon quantitative, sur des exemples simples, la question de la probabilité de trajectoires). Si l'on en reste au niveau conceptuel, le lien entre les deux formulations du second principe est discuté dans la section suivante.

\footnotetext{
${ }^{15}$ En cela, on conviendra que le second principe est un principe satisfait en probabilité, s'appliquant à des collections d'entités, et non un principe universel toujours satisfait et relatif à des parcelles de matière individuelles. Les boules de billard de la mécanique sont soumises aux mêmes perturbations que les myriades de particules du système statistique. Mais l'influence des perturbations élémentaires sur des masses plus importantes et le nombre de chocs concerné beaucoup moins important pour un même laps de temps fait que le caractère irréversible ne se fait pas sentir dans le cas du mouvement des boules de billard.
} 


\section{Le lien entre les deux formulations du second principe}

\subsection{Unité conceptuelle}

Nous sommes maintenant en effet capables de percevoir l'unité conceptuelle profonde entre les deux formulations du second principe : la formulation historique exprimant l'évolution monotone d'une fonction portant sur des quantités de chaleur échangées et des températures, et la formulation de Boltzmann exprimant l'augmentation de la probabilité des états macroscopiques. Les deux formulations renvoient à des changements dans l'espace, ce qui n'était pas évident a priori quand on exprime des fonctions telles $\int \frac{d Q}{T}$ ou $\mathrm{k}_{\mathrm{B}} \operatorname{Ln} \Omega$ en fonction du temps, où nulle variable d'espace n'apparaît.

Les variations dans l'espace des propriétés du système, motrices des changements temporels, restent implicites dans les premières expressions du second principe : mais les réservoirs avec lesquels le système échange, ou les compartiments en son sein, sont à des endroits différents et permettent de définir des gradients de température ; on retrouve ces gradients dans la production d'entropie, exprimant que l'entropie est une fonction non locale et caractérise l'homogénéité d'un système (la dérivée par rapport au temps de l'entropie est d'autant plus importante qu'il y a davantage de gradients spatiaux dans le système). Dans les deux expressions du second principe rappelées à l'instant, l'évolution naturelle ou spontanée, nous dirons encore probable, d'un système isolé $^{16}$ composé d'un grand nombre d'éléments se fait depuis un état inhomogène vers un état plus homogène. C'est la même image physique. On en a parlé explicitement à propos de la formulation statistique ; l'image fonctionne aussi dans la formulation portant sur les échanges de chaleur : en effet, dans un milieu inhomogène en température, l'homogénéité va être atteinte par le processus selon lequel les zones plus chaudes vont céder de la chaleur aux zones plus froides. Dans le titre de notre texte, nous avons parlé de «flèches » de l'espace pour parler de ces gradients à mettre en relation avec les dérivées par rapport au temps.

Si les processus physiques d'effacement des inhomogénéités se ressemblent, les variables concernées ne sont pas les mêmes dans les deux formulations. Dans le cas de la formulation macroscopique historique, il s'agit de la température, alors que dans le cas de la formulation statistique, il s'agit d'une concentration. Nous ne regarderons pas cette question en détail du point de vue des équations, mais nous considérerons que les deux formulations pourraient chacune concerner tout type de variable, c'est-à-dire non seulement la température dans le premier cas, et non seulement les concentrations dans le second. L'inhomogénéité en température peut être comprise comme une inhomogénéité de répartition des particules, une zone plus chaude étant en quelque sorte l'équivalent d'une zone plus concentrée : les particules vont plus vite et, via le lien distance-temps-vitesse, c'est comme si elles étaient plus proches les unes des autres (et, réciproquement, l'inhomogénéité en concentration a une valeur «thermique $\left.{ }^{17}\right)$. A ces inhomogénéités des paramètres, on associe ce que l'on appelle l'entropie de configuration, que nous traitons depuis le début. On définit aussi ce que l'on appelle les entropies de vibration et de rotation. Elles méritent le nom d'entropie par défaut, car l'énergie qu'elles véhiculent ne peut servir pour du travail macroscopique (cf. la définition de J. Keenan, rappelée dans [GYF 15]: l'entropie est associée à l'énergie qui ne concerne pas le travail). Les gradients d'énergie (microscopique) qui leur correspondent vont bien être des moteurs d'évolution.

\footnotetext{
${ }^{16} \mathrm{Cf}$. notre remarque à propos de ce qualificatif dans une note ci-dessus.

${ }^{17} \mathrm{C}$ 'est bien dans le sens chand $\rightarrow$ froid que va la chaleur. On peut dire réciproquement que la chaleur négative va du froid vers le chaud; la perte d'énergie dans les zones chaudes au profit des zones froides fait baisser leur température. De même dans une répartition inhomogène de particules, les particules vont des zones plus concentrées vers les zones moins concentrées (et le «manque » de particules va en sens inverse).
} 


\subsection{Lien entre les polarités dans le temps et dans l'espace; retour sur la production d'entropie}

Nous voyons donc le rôle de la variable d'espace dans les gradients et le passage d'un état inhomogène vers un état homogène. Nous avons parlé jusqu'à présent de gradients de température ou de concentration (ou plus précisément de nombres de particules en fonction de la position dans le système) ; l'examen de l'expression générale de la production d'entropie montre que les termes présents relatifs aux divers phénomènes irréversibles font tous in fine intervenir des gradients.

Pour ce qui est des réactions chimiques, il n'apparaît à première vue dans la production d'entropie ni variable d'espace ni gradients, mais des affinités chimiques et des vitesses de réaction. Variables d'espace et gradients apparaissent pourtant, c'est une question d'échelle : en dernière analyse, une affinité correspond à des différences de potentiels chimiques de molécules sises à des endroits différents, et qui vont réagir entre elles pour diminuer une énergie ${ }^{18}$. Les phénomènes dissipatifs liés à la viscosité d'un écoulement sont aussi associés aux gradients des vitesses au sein $\mathrm{du}$ fluide. Les termes de diminution de volume en relation avec une augmentation de la pression sont rangés dans le travail mais ont la même signification par rapport aux gradients.

Si l'expression «finale» de la production d'entropie montre des gradients au carré, le déroulement des calculs montre une étape intermédiaire sous la forme d'une somme de produits entre des flux et des forces ${ }^{19}$. Le lien entre ces grandeurs est présenté par Glansdorff et Prigogine [GLA 71] comme une extension du second principe : ils s'annulent en même temps ; on postule leur lien par des relations linéaires au voisinage de l'équilibre, ce qui fait apparaître des carrés. Comme le second principe exprime le passage du chaud au froid ou de l'inhomogène à l'homogène (c'est-àdire un lien nécessaire entre des gradients et des dérivées temporelles), la relation flux force est déjà une façon de le dire. In fine, les relations entre flux et forces font apparaître dans $\mathrm{P}(\mathrm{S})$ des affinités ou des gradients élevés au carré et l'on a bien dans chaque cas pris isolément une équivalence entre adoucissement de gradients et production d'entropie positive. La positivité d'un coefficient de diffusion de matière ou de chaleur en est encore une expression. Dans notre compréhension, l'écriture flux $=$ force (à un coefficient près) est une forme de degré zéro des lois physiques [GUY 12].

L'expression historique du second principe nous a orientés vers des systèmes lieu d'échanges thermiques : nous n'avons envisagé que ces phénomènes à l'exclusion des autres possibles. Auraiton défini l'entropie, aurait-on formulé le second principe de façon différente si l'on avait traité de systèmes lieu de phénomènes de diffusion de matière, de réaction chimique ou d'autres phénomènes irréversibles ? L'expression statistique est une réponse : on a écrit un principe d'évolution portant sur une organisation de la matière microscopique. Revenant à l'expression historique macroscopique, on peut dire qu'un système qui n'est lieu que d'échanges thermiques est plus simple qu'un système ouvert et pourtant susceptible d'évolution. On y fait des mesures calorimétriques elles-mêmes relativement plus simples à effectuer dans un premier temps ; cela n'empêche pas des réactions chimiques ou de la diffusion de matière, inconnues et cachées dans le système et qui vont jouer sur les valeurs annoncées des transferts $\Delta \mathrm{Q}$ mesurés et des $\mathrm{Cp}$ calculés. La quantification des

\footnotetext{
${ }^{18} \mathrm{Si}$ l'on admet que l'on ne peut séparer in fine évolution dans le temps et évolution dans l'espace, on ne peut fondamentalement séparer réaction chimique du phénomène de diffusion ; la séparation est artificielle et liée à un choix d'échelle : on homogénéise les phénomènes de transport associés à la réaction chimique supposés se dérouler à une échelle plus petite. Attention aux unités des coefficients et grandeurs dans ces opérations, à transformer d'un phénomène à l'autre en fixant des longueurs et temps caractéristiques, c'est-à-dire en choisissant une échelle d'observation.

${ }^{19} \mathrm{~L}$ 'expression historique en somme de $\mathrm{Q}_{\mathrm{i}} / \mathrm{T}_{\mathrm{i}}$ peut être comprise, pour un système donné, comme le produit d'un gradient de $1 / \mathrm{T}$ par un flux de chaleur (il suffit pour cela de diviser l'expression en 1/T par une longueur caractéristique du système et le terme $Q$ par une surface d'échange caractéristique).
} 
phénomènes peut s'affiner dans de nouvelles itérations, sachant le bouclage récursif qu'il y a de toute façon dans le choix d'une échelle de température.

\section{Seconde partie : espace, temps et thermodynamique}

A l'occasion de la formulation du second principe, de nombreux concepts et questions apparaissent sur lesquels nous voulons revenir à la lumière de notre compréhension de l'espace et du temps : l'équilibre, les échelles, la distinction entre travail et chaleur, le problème du temps, l'expression mathématique de certaines formulations, l'affectation d'une entropie à une particule individuelle, etc.

\section{Echelles de temps et d'espace}

\subsection{Travail, chaleur et entropie}

Revenons sur la distinction entre travail et chaleur. Elle n'est pas un résultat de la théorie mais est posée en préalable. Avec elle, le développement des calculs apporte un certain nombre d'informations intéressantes et d'habitude présentées comme inhérentes au second principe dans sa version macroscopique: - si on veut que, lors d'un cycle, le système fournisse du travail à l'extérieur, il lui faut échanger de la chaleur avec une source chaude et une source froide (et non avec une seule source) ; - le système reçoit de la chaleur de la source chaude et en cède à la source froide : - le rendement de Carnot est d'autant meilleur (c'est-à-dire produit le maximum de travail) que l'écart entre la température de la source chaude et de la source froide est grand.

La distinction entre chaleur et travail est donnée en somme par l'observation et est une expression de la dualité de niveaux qui fonde la thermodynamique : - le travail est associé aux mouvements à notre échelle et pertinents pour notre activité ; - la chaleur est associée à des mouvements microscopiques invisibles20. Le bouclage entre les deux niveaux est exprimé par le bilan d'énergie $\mathrm{Q} 1+\mathrm{Q} 2+\mathrm{W}=0$ et la relation de Carnot-Clausius pour un cycle $\sum \frac{Q_{i}}{T_{i}}=0$. La fonction entropie qui en dérive, ainsi que ses propriétés, intègre cette dualité d'échelle. La formule de Boltzmann $\mathrm{S}=\mathrm{kb} \ln \Omega$ permet de faire le même constat à propos de la dualité d'échelles (on regarde des situations individuelles que l'on compare au sein d'un ensemble).

$\mathrm{Si}$ on ne fait pas la séparation entre travail et chaleur, tous les mouvements des particules du système sont considérés sur le même plan. On peut encore comprendre l'évolution d'ensemble comme du transport du plus concentré vers le moins concentré, en voyant le travail comme une chaleur ordonnée, macroscopique, qui a besoin de « froid» pour se développer. Dans les systèmes qui en produisent, le travail peut être considéré comme fraction d'une quantité de chaleur totale cédée Q'1 (avec Q2 = Q'1 = Q1 + W en valeurs absolues). Le second principe encadre un inévitable gaspillage: pour assurer un mouvement macroscopique dans une seule direction $\mathrm{W}$, on ne peut éviter des mouvements parasites microscopiques dans Q1; en d'autres termes encore, il est improbable que tous les mouvements microscopiques aillent dans la même direction, correspondant à celle du travail W. Dans les raisonnements sur les échanges de chaleur et de travail, les réservoirs de chaleur avec lesquels Q1 et Q2 sont échangés ne sont a priori pas concernés par la manifestation $\mathrm{du}$ travail W. On aurait pu imaginer des effets réciproques (le travail W va-t-il contribuer à « chauffer » le milieu extérieur ?).

\footnotetext{
${ }^{20}$ A l'invisibilité est associée l'absence de maîtrise.
} 


\subsection{Peut-on parler d'entropie pour une particule individuelle ?}

L'entropie manifeste une propriété collective ou macroscopique d'un ensemble de particules microscopiques individuelles. Si on regarde le comportement d'un seul atome, on ne peut pas faire de distinction entre travail et chaleur. Parler d'entropie pour une particule isolée n'a a priori pas de sens. Certains auteurs pourtant (cf. [BER 07], [GYF 05]) définissent l'entropie de particules individuelles sans se poser la question d'échelles. Ils ont certes besoin dans leur développement de réservoirs (avec lesquels le système échange des quantités de chaleur) et de weight processes qui définissent le travail. La distinction d'échelles y est cachée : le réservoir représente l'aspect microscopique et le weight process l'aspect macroscopique. On met en évidence les limites de cette approche refusant la dualité d'échelle en posant les questions [GUY 08] : - peut-on parler d'un weight process pour un seul atome ? - peut-on regarder les mouvements de la paroi du réservoir et leur associer du travail?

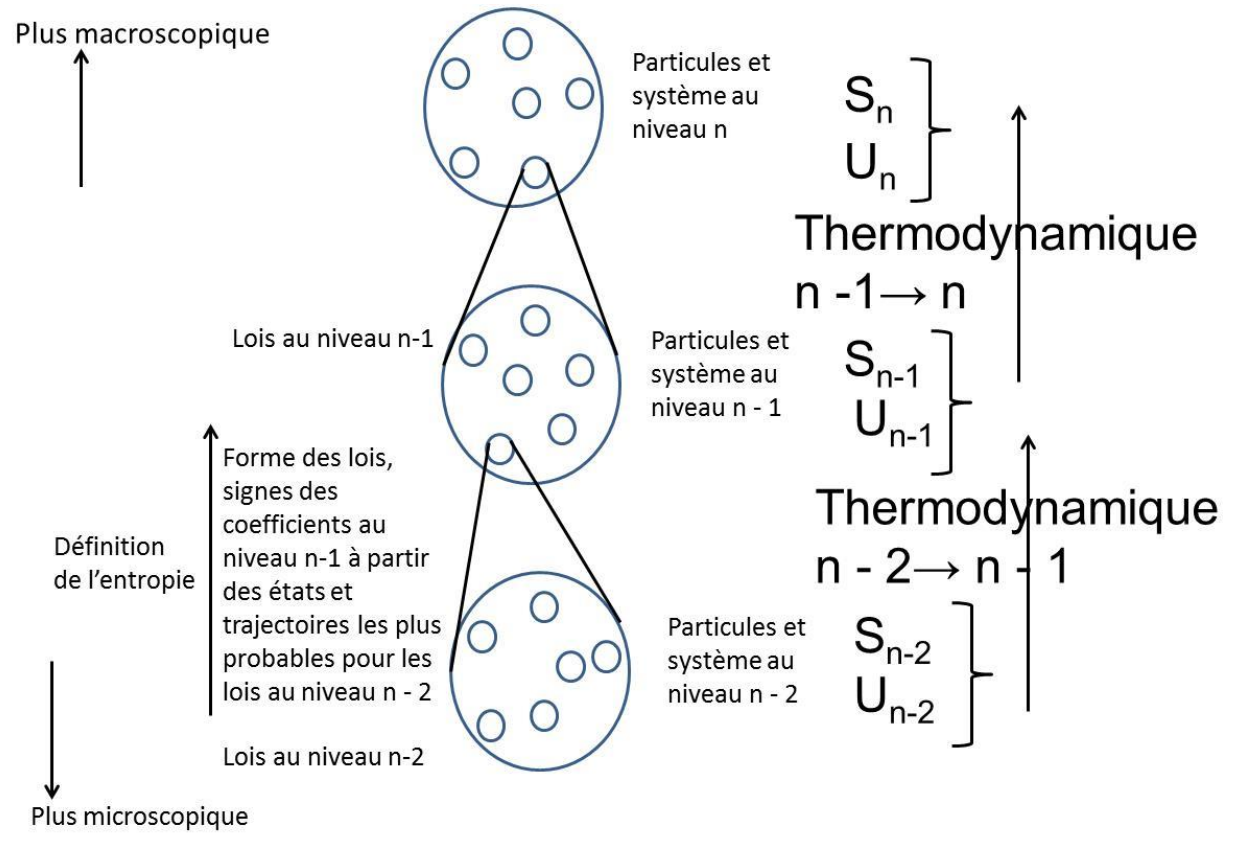

Figure 6. Hiérarchie infinie d'échelles relatives emboîtées : une échelle (ou niveau d'échelle) exprime la situation d'un couple \{petit, grand\} ou encore \{particule, système\} par rapport à d'autres couples globalement plus grands ou plus petits. Les mots « macroscopique » ou « microscopique » n'ont pas de sens en soi. Une particule vue comme un bloc inerte peut être comprise comme un ensemble de particules plus petites à un niveau inférieur. A un niveau donné (pour un couple donné) on peut définir des lois de comportement en figeant les comportements variés que l'on pourrait définir à l'intérieur des particules élémentaires à un niveau inférieur. La façon de figer ces comportements internes peut se faire en maximisant une fonction probabilité ou entropie ; cette procédure, qui permet de passer d'un niveau à l'autre peut être " oubliée "; elle se manifeste de façon résiduelle par des formes de lois et des conditions de signes portant sur les paramètres qui y interviennent au niveau étudié. Si on le souhaite on peut marquer ces différents niveaux par des notations différentes (voir texte).

Toutefois, pour aller dans le sens des auteurs précédents (au moins pour l'affectation d'une entropie à une particule isolée), il nous paraît possible d'associer une entropie à une entité individuelle : cela peut se faire sous réserve de considérer une particule même comme une famille de particules (inconnues) à l'échelle inférieure. On lui affecte une propriété unique comme à un ensemble, dont la valeur est égale à la valeur la plus probable de propriétés définies à une échelle plus petite. L'entropie quantifiant une répartition dans l'espace, la valeur choisie pour la particule 
correspond à une organisation supposée homogène des sous-particules ${ }^{21}$. La particule est alors implicitement composite mais en équilibre interne et donc à entropie maximum; on voit l'intervention d'un concept d'équilibre reliant entropie, échelle et probabilité, dans un continuum potentiellement infini d'échelles emboîtées (cf. [GUY 11b], [GUY 11c], [GUY 13a], [GUY 15] ; [SOH 12]). On pourrait ainsi, cette fois en allant vers des systèmes plus grands, se poser la question de la probabilité d'évolutions à l'échelle mégascopique pour des ensembles dont les « particules » sont des déjà des systèmes macroscopiques à l'échelle humaine (des morceaux de roche par exemple par rapport à des montagnes).

Précisons les mots utilisés : parler d'un système à l'échelle macroscopique, ne signifie pas qu'il n'y a rien de " petit ». Le système en question n'est pas pris d'un seul tenant : il est lui-même défini par de petits sous-systèmes (ou particules, ou pixels...) et intègre donc du micro et du macro. Le mot échelle (macroscopique ici) est relatif à la taille de l'ensemble du système par rapport à la taille d'autres systèmes plus petits (qui sont eux-mêmes composites, avec des «particules » plus petites encore). La pensée relationnelle fonctionne de façon implicite dans l'utilisation des mots : on n'est pas macroscopique en soi, mais toujours par comparaison avec d'autres systèmes plus microscopiques (ou moins macroscopiques). Le mot échelle est en quelque sorte composite (on pourrait parler de niveau d'échelles ?). Pour chaque échelle ou niveau d'échelle n (Fig. 6), on peut définir un ratio entre la taille de l'ensemble et la taille de la particule individuelle envisagée pour ce système. On y définit des variables $\mathrm{V}_{\mathrm{n}}$ qui ne sont pas forcément les mêmes d'une échelle à l'autre. La signification des variables d'espace et de temps est également fonction du système et pourrait être marquée par un indice $n$. Dans la compréhension que nous venons de proposer, une entropie permet de passer des grandeurs $V_{n-1}$ d'un niveau $n-1$ à celles $V_{n}$ du niveau $n$, par des moyennes probabilistes appropriées ; on pourrait la noter $S_{n-1 / n}$ ou bien savoir que $S_{n}$ signifie cet outil du passage du niveau n-1 au niveau n (il n'y a pas de raison qu'une seule fonction $\mathrm{S}$ serve de la même façon pour tous les passages d'un niveau i-1 au niveau i, sachant que la façon de faire les moyennes peut dépendre de la définition même des grandeurs concernées).

\subsection{Peut-on remplacer les écritures $d_{i} S$ et $d_{e} S$ par d'autres expressions ?}

Comme on l'a vu dans la section 2, les calculs apparaissant dans $\mathrm{d}_{\mathrm{i}} \mathrm{S}$ et $\mathrm{d}_{\mathrm{e}} \mathrm{S}$ font intervenir les mêmes termes en $\mathrm{Q}_{\mathrm{i}} / \mathrm{T}_{\mathrm{i}}$; la différence porte sur des conventions de signe. Dans le cas de $\mathrm{d}_{\mathrm{e}} \mathrm{S}$ les échanges se font avec l'extérieur; dans le cas de $\mathrm{d}_{\mathrm{i}} \mathrm{S}$, les échanges concernent des sous-parties du système. Dans les deux cas, il s'agit du même phénomène de propagation de la chaleur du chaud vers le froid. La distinction entre intérieur et extérieur du système est aussi associée à la distinction de deux échelles : le système macroscopique se définit à la fois par un extérieur avec lequel il échange de façon globale et par un intérieur ; celui-ci peut être soit pris comme un tout inerte (auquel cas $\mathrm{d}_{\mathrm{i}} \mathrm{S}=0$ ), soit compris comme composite avec distinction à nouveau de sous-parties plus « microscopiques » où se déroulent des phénomènes et faisant l'objet d'échanges. On peut continuer ce jeu de poupées russes (chaque sous-partie est un système avec son extérieur et ses échanges, et son intérieur possiblement changeant). On pourrait ainsi décaler la distinction $\mathrm{d}_{\mathrm{e}} \mathrm{S} / \mathrm{d}_{\mathrm{i}} \mathrm{S}$ en relation avec cette migration vers ces échelles plus internes du système (en utilisant des notations $S_{n}, S_{n-1}, S_{n-}$ 2 etc. comme on vient de l'indiquer), les phénomènes apparaissant les mêmes aux différents niveaux où l'on se place, la distinction portant simplement sur la taille où l'on définit un système, avec son intérieur et son extérieur.

Cela serait certainement faisable, mais il nous apparaît approprié de reprendre cette démarche en discutant les notations même utilisées : malgré leur indéniable efficacité et leur rôle conceptuel, les notations $\mathrm{d}_{\mathrm{i}} \mathrm{S}$ et $\mathrm{d}_{\mathrm{e}} \mathrm{S}$ (associées à $\mathrm{dS}$ ) nous paraissent discutables. Elles peuvent paraître en effet

\footnotetext{
${ }^{21}$ Sans prétendre à la validité complète de la comparaison, on notera que l'on se pose aujourd'hui la question de l'organisation spatiale des quarks à l'intérieur des nucléons.
} 
comme des opérations portant sur une même fonction $S$, et qui seraient différentes par le mode de différenciation. Et, une fois de plus, la variable d'espace a disparu. Dans l'esprit de notre propos, il nous semble que la distinction entre intérieur et extérieur, associée à la dualité $\mathrm{d}_{\mathrm{e}} \mathrm{S} / \mathrm{d}_{\mathrm{i}} \mathrm{S}$, pourrait être lue de façon avantageuse dans l'écriture d'une équation aux dérivées partielles de bilan, portant sur un couple \{entropie S, flux d'entropie $\mathrm{F}^{22}$ \}, et faisant apparaître un couplage entre les variations de grandeurs dans l'espace et dans le temps ; telle :

$$
\frac{\partial S}{\partial t}+\frac{\partial F}{\partial x}=0
$$

$\mathrm{Ou}$

$$
\frac{\partial S}{\partial t}+\frac{\partial F}{\partial x}=P(S)
$$

$(20$ ')

A une dimension d'espace (dans le cas général à trois dimensions, le terme de flux $\partial \mathrm{F} / \partial \mathrm{x}$ doit être remplacé par une divergence). Si l'on reprend la discussion sur les différentes échelles emboîtées auxquelles nous pouvons nous placer, la question est alors de définir le volume élémentaire représentatif, c'est à dire la distinction entre l'échelle d'observation et la quantité de matière « ultime » contenue dans le petit système local. La question associée porte sur l'existence ou non d'un terme de source qui viendrait s'ajouter aux termes précédents et jouer le rôle de production d'entropie $\mathrm{P}(\mathrm{S})$ ou $\mathrm{d}_{\mathrm{i}} \mathrm{S}$. On doit alors se demander si le petit volume représentatif sur lequel est écrite l'équation est le siège ou non de phénomènes pouvant donner lieu à un terme $\mathrm{d}_{\mathrm{i}} \mathrm{S}$. Plusieurs décisions peuvent être prises, suivant que l'on s'arrête ou non à une échelle où le volume représentatif est finalement considéré comme inerte.

Nous ferons le choix de nous arrêter à un niveau où l'équation ci-dessus n'a pas de terme de source. D'après l'analyse proposée dans les sections précédentes, l'entropie permet de passer d'un niveau d'échelle à un autre en " lissant» les phénomènes qui se déroulent à un niveau sous-jacent (en en gardant la trace la plus probable). Une fois qu'elle a joué ce rôle, l'entropie est en somme inutile ; la trace de son action se manifeste par la forme des lois du niveau supérieur, par le signe et la valeur des coefficients qui y interviennent etc. Ainsi si on connait les lois de la diffusion et les paramètres associés, il n'est pas utile d'écrire une équation portant sur l'entropie ; le signe du coefficient D, D > 0, en est la trace. Si l'on décide de définir différentes entropies Sn, Sn-1 etc. comme nous l'avons envisagé plus haut comme possible, les équations (20) et (20') précédentes engendrent aux différents niveaux de l'emboîtement d'échelles, une série d'équations du type suivant :

$$
\begin{gathered}
\frac{\partial S_{n}}{\partial t}+\frac{\partial F\left(S_{n}\right)}{\partial x}=P\left(S_{n}\right) \\
(21) \\
\frac{\partial S_{n-1}}{\partial t}+\frac{\partial F\left(S_{n-1}\right)}{\partial x}=0
\end{gathered}
$$

Ecrire un bilan entropique, avec ou non de terme de source, paraît une étape intermédiaire d'un processus de mise en équation. Si on dit $\mathrm{P}(\mathrm{S}) \neq 0$ ou si on écrit une inégalité faisant intervenir $\mathrm{S}$,

\footnotetext{
${ }^{22}$ L'expression de « flux d'entropie » est utilisée dans les bilans généraux écrits sur l'entropie. Cette expression semble augmenter le mystère qui entoure la grandeur, mais c'est en somme une métonymie signifiant que les apports de matière dans un système ouvert véhiculent des inhomogénéités.
} 
c'est qu'il y a des phénomènes à une échelle inférieure que l'on n'a encore pas lissés. $\mathrm{S}$ va nous servir de mesure pour cela. On retrouve ce qui se passe ailleurs en physique où forme des lois et signes des coefficients peuvent être compris à partir de conditions d'extremum imposant un signe à des fonctionnelles plus générales (on pourrait supposer que, là aussi, cela dérive de phénomènes cachés à un niveau inférieur que l'on a lissés); les principes extrémaux en physique (à l'intérieur desquels on peut comprendre les lois physiques) jouent le même rôle que le second principe (cf. [BAS 02]). On pourrait se demander pourquoi la thermodynamique s'est fait remarquer de façon spéciale dans un précepte tel que le second principe?

Dans le cas où plusieurs phénomènes se déroulent, chacun contribuant au développement d'hétérogénéités, l'écriture de la production d'entropie complète permet de discuter leurs liens à cet endroit (nous avons pour l'instant parlé des phénomènes comme agissant de façon séparée). Sans parler de leur forme, l'existence des relations d'Onsager, en est l'expression ${ }^{23}$.

\section{L'équilibre et les variables d'espace et de temps}

La notion d'équilibre fait intervenir de façon plus ou moins cachée des choix portant sur les variables d'espace et de temps. La première définition concerne des systèmes dont les propriétés ne varient pas avec le temps. La question qui se pose alors est de savoir sur quelle durée ils sont observés : la signification n'est pas la même si c'est pendant quelques femto-secondes ou quelques milliards d'années! L'équilibre est aussi un autre nom de l'homogénéité : si le système est homogène, il n'évolue pas. Cela nous ramène à la variable d'espace et son échelle : suivant la finesse du découpage, on peut ou non décréter une homogénéité. Les notions d'équilibre ou de déséquilibre ne sont donc pas substantielles mais dépendent de choix «arbitraires » d'échelles de temps et d'espace qui nous intéressent. C'est-à-dire, selon des considérations plus fondamentales encore (comment définir l'espace et le temps ?) de la coupure que nous faisons (toujours en fonction de nos besoins particuliers) entre ce que nous décrétons comme mobile (sur quoi nous définissons le temps) et ce que nous décrétons comme immobile (sur quoi nous définissons l'espace ; cf. nos travaux). On boucle donc le cycle de récursivité en reconnaissant qu'espace et temps n'ont pas de définition extérieure aux systèmes et sont effectivement appuyés sur la matière et ses mouvements. La possible stationnarité d'un système (son invariance en fonction du temps) suppose que l'on n'étudie qu'une partie du monde ; on a laissé de côté ce qui est mobile (et permet de définir le temps...).

Revenons-en à l'entropie. Certains auteurs affirment qu'il faut l'équilibre pour la définir, d'autres non. Si l'on regarde la définition statistique $\mathrm{S}=\mathrm{k}_{\mathrm{b}} \ln \Omega$, on semble a priori pouvoir se passer de toute notion d'équilibre. Mais on ne s'affranchit pas de choix d'échelles d'espace ni de temps, car il faut encore préciser sur quelle période de temps et quelle amplitude d'espace on regarde les configurations microscopiques? Si l'on regarde la définition historique de l'entropie par des échanges de chaleur, on lit couramment que l'équilibre est requis, ce qui nous ramène à la discussion sur les échelles d'espace et de temps; en fin de compte, on voit l'impossibilité d'une définition qui se passe de conventions «arbitraires ». L'indépendance d'une définition par rapport aux échelles d'espace et de temps est aussi impossible à cause de l'existence d'états métastables. Une autre façon de définir l'équilibre «vrai » est en effet de requérir la condition d'extremum global d'un potentiel thermodynamique; il n'est pas atteint dans les états métastables: une récursivité apparaît alors avec les conditions garantissant l'équilibre car, précisément, pour définir les fonctions thermodynamiques qui vont servir de potentiels (et donc définir l'équilibre), nous avons besoin de parler d'équilibre et de faire la part des dissipations qui ne répondent pas à cette

\footnotetext{
${ }^{23}$ On peut comprendre intuitivement que les gradients des différentes grandeurs influent les uns sur les autres : les chocs entre atomes interviennent de façon collective, quelle que soit leur nature.
} 
notion. Certains auteurs (par exemple[GYF 05] ; [BER 07]) insistent pour dire que leur définition de l'entropie (dans des formulations macroscopiques non statistiques) n'exige pas l'équilibre. Leur développement demande tout de même l'équilibrage des systèmes avec des réservoirs : en fait, encore une fois, les questions d'échelle de temps et d'espace y sont cachées. L'équilibre se manifeste aussi comme condition pratique pour faire des expériences de calorimétrie qui aient un sens. Quand on parle de non équilibre dans ces situations expérimentales, c'est qu'il y a des gradients spatiaux (et donc temporels) significatifs par rapport aux échelles d'intérêt.

En bref, l'équilibre se définit lorsqu'il n'y a pas de déséquilibre, et le déséquilibre (on parle de façon équivalente de dissipation, facteurs d'irréversibilité etc.) lorsqu'il n'y a pas d'équilibre, à choix d'échelle d'espace et de temps donné !

\section{L'entropie des philosophes, l'entropie des physiciens, l'entropie des autres...}

Les diverses formulations du second principe, admises sans critique et reprises abondamment par les philosophes, ont conduit l'esprit vers des fausses pistes, ou, à tout le moins, vers une conception mystérieuse de l'entropie, du temps et sa flèche, indépendante de l'espace ; on aurait envie de dire indépendante du monde ! Il est utile pour comprendre le principe de faire intervenir l'espace et les chemins qui le traversent. Il est oublié (ou homogénéisé) dans un énoncé purement temporel. L'augmentation de l'entropie est intimement, fondamentalement, structurellement, liée à la variable d'espace. Le sens du temps se fait dans la direction qui adoucit les gradients spatiaux ; l'évolution est guidée par une polarité de l'espace... Pour des systèmes macroscopiques, la flèche du temps est une flèche de l'espace, c'est une polarité de l'espace microscopique caché ${ }^{24}$. On pourra aussi utiliser le pluriel (des flèches), car les gradients multiples à l'intérieur d'un système engendrent des évolutions locales multiples permettant une multitude de temps. On attache au système un temps macroscopique unique par des conventions sur lesquelles nous ne revenons pas (voir nos travaux). On peut «projeter » la flèche du temps macroscopique sur la représentation du temps à l'échelle microscopique en décidant que le mobile marquant le temps ne revient pas en arrière et permet par sa polarité de parler de renversement éventuel du mouvement des autres particules (voir [GUY 04]). On peut dire inversement, via le lien avec les gradients d'espace, que l'orientation de l'espace (la flèche de l'espace exprime aussi la distinction entre le près et le lointain) peut être appuyée sur la flèche du temps ${ }^{25}$.

Devant la difficulté de saisir la formulation historique du second principe, certains auteurs plaident pour son oubli et proposent d'exposer d'emblée la formulation statistique dont tout le reste du formalisme se déduit (Gonczi, op. cit.). La démarche d'ensemble est alors très cohérente et rigoureuse du point de vue mathématique, comme peuvent la proposer des formulations purement axiomatiques de la thermodynamique évitant les inconvénients des deux formulations (sans prétention aucune à l'exhaustivité, voir [TIS 66]; [LEM 99]; [BON 11]; suivant les cas la formulation statistique y est encore reliée). Malgré cela, ces diverses expositions manquent de supports imagés pour la pensée. Le maintien d'un lien avec la mécanique nous parât important pour ce faire.

\footnotetext{
${ }^{24}$ Cette interprétation de l'irréversibilité du temps comme liée à des gradients, eux même liés à des conditions initiales particulières, peut se dire dans le cadre du big-bang lui-même : il impose un méga-gradient (ce n'est plus microscopique !) entraînant une polarité à toute l'histoire de l'univers (attention alors à la composer avec le caractère non extensif des grandeurs utilisées).

${ }^{25}$ La correspondance entre flèche de l'espace et flèche du temps se comprend aussi bien au niveau local dans sa multiplicité, qu'au niveau global ou statistique (où l'on s'en tient alors à un temps et un espace collectifs). C'est essentiellement dans ce second sens que l'on parle de flèche du temps, bien que, par extension, on puisse en parler pour un mouvement singulier qui n'a qu'un sens à la fois!
} 
Nous n'avons pas parlé d'autres théories faisant appel au concept d'entropie (sans lien direct avec un «second principe ») telle celle de l'information selon Shannon. Cette approche à l'intérêt de montrer le découplage entre un principe d'évolution et la description statistique d'un système ; elle montre aussi la compréhension de l'entropie comme liée au manque d'information sur sa connaissance complète. Le second principe n'est pas par lui-même un moteur d'évolution (ce sont les lois «mécaniques » que nous écrivons qui ont ce rôle), mais il encadre lois et paramètres les plus probables (cf. [JAY 57a], [JAY 57b]). Les gradients dont nous avons souligné l'importance (où sont espace et temps dans la théorie de l'information ?) sont alors des gradients de probabilité dans les messages discutés par cette théorie (il s'agit des probabilités des différents caractères du langage utilisé pour transporter l'information). Sans rentrer dans le détail des formulations mathématiques, on conviendra que, en envisageant l'information «toute seule », sans support de matière ni contrepartie dans l'espace ni le temps, on pousse jusqu'à l'absurde un processus d'abstraction ; nous ne nions pas les bonnes recettes auxquelles cela conduit, avec le risque d'aboutir à des formulations à la limite vides de sens.

\section{Le problème du temps}

Revenons sur le «problème du temps » déjà évoqué partiellement : pourquoi, à partir les lois réversibles de la mécanique, aboutit-on aux lois irréversibles de la thermodynamique ? Comme nous l'avons vu, la réponse à cette question passe par une compréhension macroscopique probabiliste : lors de l'évolution d'un système composé d'un grand nombre de particules individuelles, les perturbations de tous types agissant sur elles conduisent le système vers l'état le plus probable (elles l'écartent des trajectoires improbables). Les perturbations peuvent avoir un sens physique classique et être de multiples origines ; elles peuvent être plus fondamentalement comprises comme liées à notre méconnaissance de la valeur des paramètres d'espace et de temps dans les repères utilisés (la mécanique quantique, bien qu'elle considère a priori temps et espace comme fournissant un cadre extérieur pour les systèmes étudiés, intervient d'un point de vue semblable mais plus indirect, via les incertitudes sur les positions). La solution proposée est ainsi en prolongement de la solution probabiliste de Boltzmann. Le second principe peut être violé, il n'est pas un principe universel mais a une portée «pratique » (voir par exemple [BAL 94] ; [RIT 03]).

D'autres auteurs se sont penchés sur cette question et ont proposé des solutions différentes. Pour Balian (op. cit.), parmi de nombreux autres, la non-réversibilité effective est simplement liée au temps de retour, infiniment grand, à la disposition initiale d'un système soumis à variation. Ce résultat renvoie au théorème de récurrence de Poincaré ; mais ce dernier s'applique à des systèmes infinis répondant à la limite thermodynamique et ceci met de toute façon cet argument sur un plan différent de celui de notre approche rappelée ci-avant. Prigogine [PRI 80], [PRI 95], [PRI 96]) (cf. aussi [PET 93]) refuse quant à lui les différentes solutions que nous venons d'évoquer, cherchant une irréversibilité « intrinsèque » qui ne doive rien à une connaissance imparfaite ou limitée dans le temps. Mais cet auteur considère aussi de larges systèmes («large Poincaré systems »), conformes à la limite thermodynamique infinie et qui l'éloignent de la simplicité intrinsèque et originelle de l'irréversibilité du temps nu qu'il postule (voir l'analyse proposée dans [GUY 07]).

Dans ces diverses recherches, les auteurs poussent jusqu'à l'absurde la séparation du temps par rapport au monde. Quel sens pourrait avoir un renversement du temps tout seul ? Le temps est appuyé sur la matière, ses grains et leurs mouvements. Lorsque l'on parle d'expérience de renversement du temps, c'est bien la vitesse des particules du système que l'on inverse. Le temps est fabriqué par les lois qui combinent espace et temps : un temps réversible par celles de la physique microscopique, un temps irréversible par celles de la thermodynamique macroscopique. Il n'y a pas plus de temps objectif dans un cas que dans l'autre. La mise en regard des deux domaines nous montre que réversibilité et irréversibilité ne sont pas intrinsèques, mais pensées ensemble ; leur 
frontière est en somme choisie arbitrairement et dépend des échelles de temps, espace et nombre de particules qui nous intéressent.

Cette discussion peut être affinée en tenant compte des considérations sur les échelles données plus haut: dire que le second principe est violé, c'est se placer, non au niveau de l'échelle $\mathrm{n}$ qui nous intéresse (et pour lequel on définit l'entropie $S_{n}$ ), mais au niveau $n-1$ pour lequel on $n$ 'a pas encore fait de choix, précisément à l'aide de $S_{n}$, entre les différentes trajectoires et états plus ou moins probables. On pourrait ainsi dire qu'il y a une infinité de « seconds principes », (et définir plusieurs flèches du temps), tout dépend du niveau d'échelle où l'on se situe. Mais on peut dire aussi que le second principe exprime une qualité générique de systèmes composés de myriades de particules élémentaires et pour lesquels on cherche un comportement d'ensemble probable, quel que soit le niveau d'échelle où l'on se situe (les raisonnements sur la dispersion des particules sont valables quelle que soit leur taille).

\section{Conclusions}

\subsection{Vers de nouvelles formulations?}

Avant de conclure, regardons quelques pistes de recherche envisageables. D'une façon générale comme nous l'avons dit, il faut privilégier au départ en physique les formulations qui associent étroitement les variables d'espace et de temps (« équations de degré zéro »). Celles-ci portent sur des grandeurs physiques définies par paires, et dont on ne peut affirmer que des variations couplées. Ainsi, si on s'intéresse à la grandeur entropie $S$, on doit lui associer une autre grandeur F, que nous avons appelée flux d'entropie. On ne connaîtra que des lois du type :

$$
\frac{\partial S}{\partial t}+\frac{\partial F}{\partial x}=0
$$

Ecrites avec une seule variable d'espace (la pleine portée de ces formulations apparaît avec des couples de variables $(\mathrm{x}, \mathrm{y}, \mathrm{z})$ d'un côté, $\left(\mathrm{t}_{\mathrm{x}}, \mathrm{t}_{\mathrm{y}}, \mathrm{t}_{\mathrm{z}}\right)$ de l'autre, ces trois dernières variables servant de support à la définition d'un scalaire temps t). Si l'on attribue à $\mathrm{S}$ le sens d'une fonction de probabilités d'états, ne faut-il pas attribuer à $\mathrm{F}$ celui de probabilité de trajectoires ? Etat et trajectoire ne peuvent être fondamentalement séparés dans une compréhension où temps et espace sont construits de façon associée à partir des phénomènes eux-mêmes. Si l'on exige pour d'autres grandeurs de suivre un tel fonctionnement couplé (il faut le faire de façon générale pour tous les couples $(\mathrm{X}, \mathrm{J}),(\mathrm{c}, \mathrm{J}),(\mathrm{E}, \mathrm{p}),(\mathrm{S}, \mathrm{F})^{26}$; l'archétype de tels couples est fourni par la paire $(\mathrm{E}, \mathrm{B}) \mathrm{de}$ l'électromagnétisme vérifiant les équations de Maxwell), on traitera par exemple des couples (c, J) ou $\{$ concentration, flux $\}$ dans des lois de conservation du type :

$$
\frac{\partial c}{\partial t}+\frac{\partial J}{\partial x}=0
$$

Les deux équations écrites (22) et (23) doivent être compatibles du point de vue de leur définition des variables d'espace et de temps (et le ratio de ces dernières ; c'est en somme une nécessité de raccord même entre variables d'espace et de temps). Elles le sont si on remplit des conditions du type :

$$
\frac{\partial S}{\partial c}=\frac{\partial F}{\partial J}
$$

\footnotetext{
${ }^{26}$ On a reconnu les couples des forces $\mathrm{X}$ et des flux J, de l'énergie $\mathrm{E}$ et de la quantité de mouvement $\mathrm{p} .$.
} 
Ces fonctions expriment l'équilibre local, traduisant le lien entre deux échelles, ici entre ce qui est transporté et ce qui concerne le petit système local. Elles permettent de se ramener à une seule équation (en multipliant les deux membres de (23) par les conditions de compatibilité (24) on obtient (22)) et d'oublier par exemple la fonction entropie : elle aura servi dans la sélection des lois et paramètres à un certain niveau. Cela rejoint la formulation des mathématiciens des problèmes hyperboliques qui définissent, non pas une entropie, mais un couple \{entropie, flux d'entropie\} assorti de diverses contraintes. Les conditions dites d'entropie, permettent de sélectionner la solution physique, parmi les solutions faibles du problème posé en termes de distribution, et faisant apparaître de possibles chocs.

Le progrès dans ces formulations devra utiliser le formalisme des intégrales de chemin («path integrals »), developpé en particulier par Feynman et Wiener. Il s'agit là de calculer des intégrales portant sur des fonctions (et non sur des nombres) représentant dans notre cas les diverses trajectoires possibles, afin de déterminer les chemins optimaux. La détermination des états (fonction entropie) et des trajectoires y conduisant (intégrale de chemin) nous paraissent indissociables ${ }^{27}$. Dans notre compréhension conjointe de l'espace et du temps en effet, la définition d'un état, sur un intervalle de temps même limité, ne peut se passer d'un espace avec lequel il est en relation, c'est-àdire encore à travers lequel on le connaît : cela se traduit par des équations élémentaires de type (20) ou (23) où les grandeurs interviennent par couples, espace et temps étant en somme sur le même plan que les autres. Ainsi, nous le soulignons, il faut voir la recherche des états et celle des trajectoires comme deux aspects du même problème: on peut comprendre intuitivement qu'un faisceau de trajectoires divergentes (extrémum d'une intégrale de chemin) va conduire à des états plus également distribués (plus haute entropie) que dans le cas où le faisceau reste groupé (sans compter le fait que le calcul même d'une intégrale de chemin doit tenir compte des points de départ et d'arrivée d'une évolution). Des auteurs comme Dill [DIL 08] ou Kikuchi [KIK 66] se sont intéressés à la détermination des trajectoires sans insister comme nous le faisons, sur l'unité conceptuelle à chercher $\mathrm{ici}^{28}$. Nous ne retenons pas sur ce sujet son abord par l'équation de Liouville (flots dans l'espace des phases) qui, d'une part, n'envisage pas les trajectoires dans l'espace physique en tant que telles, et, d'autre part, repose sur une propriété de réversibilité non possédée pas les routes soumises aux perturbations dont nous avons parlé. Le lien avec les intégrales de chemin mérite certes d'être explicité.

Cette approche devra être précisée ; elle doit permettre d'éviter les formulations $d_{i} S$ et $d_{\mathrm{e}} S$ dont on a souligné les difficultés et se placer d'emblée en système ouvert. En relation avec elle, il faudra reprendre la question des notations portant sur les diverses fonctions utilisées selon les échelles étudiées, comme on l'a montré dans la section 6.2. On peut voir une certaine incohérence dans l'écriture d'un bilan entropique au niveau n faisant apparaître un terme de source. Celui-ci en effet fait implicitement intervenir des variables au niveau $n-1$ qui sont celles des sous parties du système ; elles ne sont pas forcément les mêmes que les variables optimisées du niveau d'ensemble n qui interviennent par exemple dans le terme de flux (on aura noté plus haut par exemple les notations différentes pour les échanges de chaleur interne $\mathrm{Q}_{\mathrm{ij}}$ et les échanges avec l'extérieur $\mathrm{Q}_{\mathrm{i}}$ ). L'utilisation de notations mathématiques différentielles (que ce soit dans l'écriture de type $\mathrm{dS}, \mathrm{d}_{\mathrm{i}} \mathrm{S}$, $\mathrm{d}_{\mathrm{e}} \mathrm{S}$, ou dans celle utilisant les dérivées partielles $\partial \mathrm{S} / \partial \mathrm{t}$ ou $\partial \mathrm{F} / \partial \mathrm{x}$ ) laisse aussi supposer que l'on peut effectuer des passages à la limite pour des systèmes de taille tendant vers zéro. Cela n'est pas compatible avec le maintien de termes de source qui supposent des phénomènes se déroulant à une

\footnotetext{
${ }^{27}$ Rappelons notre propos de départ (section 4): la question n'est pas seulement de déterminer un état optimal mais de comprendre pourquoi le système y va effectivement.

${ }^{28}$ Tout en notant la similitude des hypothèses et des démarches faites dans les deux cas (états / trajectoires). On peut penser que l'approche discutée ici doit permettre de relier de façon organique le principe d'extrémum de la production d'entropie et celui de l'entropie.
} 
échelle plus petite encore. C'est aussi dire que l'espace et le temps sont alors définis à une certaine échelle et n'épuisent pas leur définition à une échelle plus petite. Une véritable équation de degré zéro (sans terme de source) représente un cas limite où espace et temps s'appuient sur des grains de matière dont on ne cherche plus le caractère composite ou non.

\subsection{Thermodynamique et autres théories physiques}

L'intérêt d'une formulation portant sur une paire telle \{entropie, flux d'entropie\} en fonction de la paire $\{r, t\}$ est son invariance de Lorentz. C'est une condition élémentaire pour associer thermodynamique et relativité. Dans la mécanique quantique elle-même, on doit privilégier une formulation du même type, invariante de Lorentz : l'équation de Schrödinger n'est pas satisfaisante de ce point de vue, il faut s'orienter vers des équations de type Dirac, avec deux fonctions probabilistes en dualité $\Psi$ et $\Phi$. Les localisations précises sont remplacées par des fonctions probabilistes. La thermodynamique a aussi un sens probabiliste. La question se pose de savoir si, dans le traitement des aspects probabilistes, nous devons ajouter les deux formulations thermodynamique et quantique, ou d'une façon ou d'une autre, les identifier en rediscutant le sens donné aux différentes fonctions? A première vue, les sens des probabilités ne sont pas les mêmes : par rapport à l'espace dans le cas de la mécanique quantique, par rapport à des ensembles d'états (ou de trajectoires vues comme telles) pour la thermodynamique. Mais si l'on veut bien voir derrière l'état le plus probable (auquel nous attachons une fonction $\mathrm{S}$ ) une probabilité de différents états microscopiques dans l'espace, le lien entre les deux approches devient possible. On pressent ainsi une façon d'unifier la quantification en mécanique quantique et le choix des états en thermodynamique comme la manifestation du saut d'états ou de trajectoires non probables à une certaine échelle ${ }^{29}$.

\subsection{Encore une formulation du second principe}

En conclusion, revenons sur une formulation du second principe de la thermodynamique qui tienne compte de notre analyse. Elle concerne des systèmes isolés macroscopiques, c'est-à-dire composés d'un très grand nombre de particules individuelles, et dotés de la qualité d'extensivité ; c'est-à-dire que les propriétés du système sont en proportion des propriétés des sous-systèmes de tailles quelconques que l'on peut y définir : il n'y a pas de forces à longue portée. Pour de tels systèmes nous disons que l'évolution temporelle se fait dans le sens d'un adoucissement des gradients spatiaux qui existent en leur sein.

Ceci signifie en particulier que :

la chaleur va du chaud vers le froid ; une partie de cette chaleur, mais non toute, peut être distraite pour produire du travail ;

le système évolue vers des états plus probables, à condition de définir des probabilités pour les distributions dans l'espace des particules individuelles et pour les trajectoires y conduisant.

L'entropie est la fonction qui assure que, dans un tel système, la chaleur va bien du chaud au froid, ou encore que la distribution statistique de la répartition des entités individuelles évolue du moins probable vers le plus probable, ou du plus ordonné vers le moins ordonné. Cela correspond à une évolution depuis des états d'énergie inhomogène vers des états d'énergie plus homogène (ou

\footnotetext{
${ }^{29}$ La modélisation des phénomènes physiques et le repérage de l'espace et du temps sont tout un. Les probabilités associées aux phénomènes ne peuvent être traitées indépendamment de l'espace et du temps qui fournissent une distribution de probabilité uniforme comme base d'autres distributions (pensée relationnelle). La probabilité de telle propriété correspond à la proportion des particules ayant cette propriété, dans l'espace physique associé au système dans son ensemble. On peut comprendre ainsi l'usage des probabilités en mécanique quantique : un saut quantique est un saut par-dessus des niveaux improbables ; de la même façon, le second principe est un principe de sélection (à un niveau plus macroscopique, on ne voit pas les trajectoires improbables).
} 
encore depuis des états plus réactifs vers des états moins réactifs etc.). L'entropie est ainsi une fonction associée au transfert ou à la dissipation de l'énergie. C'est une fonction caractérisant l'homogénéité d'un système et ne peut se passer de variable d'espace pour sa compréhension. Elle a un caractère abstrait : pour l'efficacité de la pensée, on l'a détachée artificiellement des objets du monde. Il convient alors de ne pas la « réaliser » c'est-à-dire en faire une substance autonome (ni elle ni l'énergie). Dans la mesure où, à l'échelle de l'univers, des forces à longue portée (en l'occurrence les forces de gravitation) s'expriment, on ne peut plus dire avec Boltzmann que l'entropie de l'univers augmente (la formation des galaxies et des étoiles nous montre au contraire l'exemple de création d'hétérogénéités), même si l'on parle toujours de la constance de l'énergie de l'univers.

Le second principe perd un peu de son mystère. Il est compréhensible en termes d'espace, temps, et mouvement. Il est libéré de sa compréhension historique dans ce qu'elle pouvait avoir de particulier. Le sens du temps macroscopique est lié à l'évolution vers l'homogénéité ; il a un sens statistique et sa polarité n'est pas en contradiction avec la réversibilité des chemins microscopiques décrits par la mécanique. On peut dire de façon synthétique que, pour un petit domaine de l'espace, la flèche du temps est en relation étroite avec la flèche de l'espace, c'est-à-dire avec les gradients des paramètre ; temps et espace sont tous deux appuyés sur la matière et ses grains et les relations spatiales et les relations temporelles sont les mêmes relations.

\section{Remerciements}

L'auteur remercie tous ceux dont il a lu les travaux, et toutes les personnes avec qui il a discuté de vive voix les questions abordées dans le présent article : les enseignants de thermodynamique des écoles des mines (en particulier de Paris et Saint-Etienne) et de l'université de Bruxelles, et les divers chercheurs des JETC (Journées Européennes de Thermodynamique Contemporaine, ou Joint European Thermodynamics Conference), et du congrès «Meeting the entropy challenge » (Boston, Massachussets, 2007). Nous voulons citer en particulier: B. Andresen, R. Balian, G.P. Beretta, O. Bonnefoy, D. Fargue, M. Feidt, F. Fer, C. Jallut, J. Le Coze, G. Nicolis, I. Prigogine, D. Tondeur. Merci à M. Feidt et S. Kiesgen de Richter pour leurs remarques critiques et constructives sur une version antérieure du manuscrit. Une première version de ce texte est paru dans [GUY 15b]. Les concepts sous-jacents à la présente étude sont exposés de façon générale dans un ouvrage récent [GUY 19].

\section{Références bibliographiques}

[BAL 94] BALIAN R. «Le temps macroscopique», in : Le temps et sa flèche, E. Klein et M. Spiro éd., Editions Frontières, 155-212, 1994.

[BAR 02] BARBEROUSSE A. La mécanique statistique, de Clausius à Gibbs, Belin, Paris, 240 p., 2002.

[BAS 02] BASDEVANT J.-L. Principes variationnels et dynamique, Cours de l'Ecole polytechnique, 102 p., 2002.

[BER 07] BERETTA G.P. « Axiomatic definition of entropy for non-equilibrium states », JETC IX, (Joint European Thermodynamics Conference), Ecole des Mines de Saint-Etienne, 24-29, 2007.

[BOL 95] [BOL 98] BOLTZMANN L. Leçons sur la théorie des gaz, Editions J. Gabay, Paris, première partie, 204 p. ; seconde partie, 280 p., 1895, 1898 [1987].

[BON 11] BONNEFOY O. Introduction à la thermodynamique classique, Cours Ecole des Mines de Saint-Etienne, 100 p., 2011.

[BRI 95] BRICMONT J. « Science of chaos or chaos in science ?» Physicalia Mag.17, 3-4, 159-208, 1995.

[CAR 24] CARNOT S. Réflexions sur la puissance motrice du feu et sur les machines propres à développer cette puissance, Bachelier, Paris, 120 p., 1824.

[CLA 64] Clausius R. Théorie mécanique de la chaleur ; traduction F. Folie, 1868, Eugène Lacroix éditeur, Paris ; édition Jacques Gabay, Paris, 1991 ; 307 p., 1864.

(C) 2020 ISTE OpenScience - Published by ISTE Ltd. London, UK - openscience.fr 
[DIL 08] DILL K.A. «The second law and biology », in : Meeting the entropy challenge, G.P. Beretta, A.F. Ghoniem, G.N. Hatsopoulos editors, American Institute of Physics, 90-110, 2008.

[FER 70] FER F. Thermodynamique macroscopique, Tome I, Systèmes fermés, Gordon et Breach, 288 p., 1970.

[GIB 02] GIBBS J.W. Elementary principles of statistical mechanics, rééd.[1965], Dover, New York, 1902.

[GLA71] GLANSDORfF P. et PRIGOGINE I. Structure, stabilité et fluctuations, Masson, Paris, 288 p., 1971.

[GON 08] GONCZI G. «La thermodynamique est-elle compréhensible ? Bulletin de l'union des physiciens, vol. 102, $\mathrm{n}^{\circ} 908$ (2) cahier enseignement supérieur, 113-122, 2008.

[GUY 04] GUY B. «L'irréversibilité vient avec le temps », in: L'éclair et le tonnerre, promenades entre l'espace et le temps, Editions EPU, Paris, 73-79, 2004.

[GUY 07] GUY B. «Prigogine and the time problem: a dialog between physics and philosophy », JETC IX (Joint European Thermodynamics Conference), Ecole des Mines de Saint-Etienne, 90-91, 2007.

[GUY 08] GUY B. " Particles, scale, time construction and the second law of thermodynamics », Meeting the entropy challenge, An international thermodynamics conference in honor and memory of Professor Joseph Henry Keenan, The MIT, Cambridge, MA, USA, October 4-5, 2007. The American institute of Physics, p. 174-179, 2008.

[GUY 11a] GuY B. «Penser ensemble le temps et l'espace », Philosophia Scientiae, 15, 3, 91-113, 2011.

[GUY 11b] GUY B. L'architecture de la thermodynamique et ses développements futurs, <hal-00611861>, 2011.

[GUY11c] GUY B. La thermodynamique, science du changement d'échelle, in [BON 11], 3-6, 2011.

[GUY 12] GUY B. Degré zéro des lois physiques, considérations heuristiques, <hal-00723183>, 2012.

[GUY 13a] GUY B. The architecture of thermodynamics and its future developments, <hal-00863970>, 2013.

[GUY 13b] GUY B. Relier la mécanique quantique et la relativité générale ? Réflexions et propositions. <hal00872968>, 2013.

[GUY 14] GUY B. Pour un nouveau paradigme. La dichotomie conceptuelle entre espace et temps est (devenue) un obstacle aux progrès de la pensée. Commençons par le mouvement ! <hal-01061765>, 2014.

[GUY 15a] GuY B. «Space, time and thermodynamics », 13th Joint European Thermodynamics Conference, Nancy, ENSIC, 238-240, 2015.

[GUY 15b] GUY B. Flèches du temps et de l'espace, une compréhension du second principe de la thermodynamique $<$ hal-01223419>, 2015.

[GUY 16] GUY B. L'espace, le temps et l'entropie, Editions Universitaires Européennes, 110 p., 2016.

[GUY 17] GUY B. What can a better coupling between space and time concepts bring to thermodynamics < hal$01529570>, 2017$.

[GUY 19] GUY B. ESPACE = TEMPS. Dialogue sur le système du monde. Penta Editions, Paris, 232 p., 2019.

[GYF 05] GYFTOPOUlOS E.P. et BERETTA G.P. Thermodynamics, foundations and applications, Dover, 756 p., 2005.

[JAN 96] Jancovici B. Thermodynamique et physique statistique, Nathan, Paris, 128 p., 1996.

[JAY 57a] JAYNES E.T. «Information theory and statistical mechanics », Physical Review, 106, 4, 620-630, 1957.

[JAY 57b] JAYNES E.T. «Information theory and statistical mechanics II », Physical Review, 108, 2, 171-190, 1957.

[KIK 66] KIKUCHI R. « The path Probability Method », Supplement of the Progress of Theoretical Physics, 35, 64 p., 1066.

[LEC 10] LE COZE J. «Le passage du macroscopique au microscopique, de la grosse forge à l'entropie statistique, quelles sont les hypothèses invisibles »; Actes des Ateliers sur la contradiction, coordination B. Guy, Presses des mines, Paris, 103-111, 2010.

[LEM 99] LemarChand H., GuYot F., JousSet L., Jullien L. Thermodynamique de la chimie, Hermann, Paris, 424 p., 1999.

[PET 93] Petrosky and Prigogine «Poincaré resonances and the limits of trajectory dynamics », Proc. Natl. Sci. USA, 90, 9393-9397, 1993.

[PRI 80] PRIGOGINE I. From being to becoming, Freeman, New York, 1980. 
[PRI 95] PRIGOGINE I. « Why irreversibility? The formulation of classical and quantum mechanics for non integrable systems », International journal of bifurcation and chaos, 5, 1, 3-16, 1995.

[PRI 96] PRIGOGINE I. La fin des certitudes, Odile Jacob, Paris, 1996.

[RIC 00] RICHET P. Les bases physiques de la thermodynamique et applications à la chimie, Belin, Paris, 464 p., 2000.

[RIT 03] RITORT F. «Work fluctuations and transient violations of the second law : perspectives in theory and experiment », Séminaire Poincaré, 2, 63-87, 2003.

[SOH 12] SOHRAB S. « On a scale invariant model of statistical mechanics, kinetic theory of ideal gas, and Riemann hypothesis », American Institute of Aeronautics and Astronautics, Nashville meeting, 52 p., 2012.

[TIS 66] TISZA L. Generalized thermodynamics, The MIT press, Cambridge, Massachussets, 384 p., 1966.

[TSA 95] TSALLIS C., «Some comments on Boltzmann-Gibbs statistical mechanics », Chaos, Solitons and Fractals, 6, 539-559, 1995. 